\title{
Reverse Approximation of Energetic Solutions to Rate-Independent Processes
}

\author{
Alexander Mielke and Filip Rindler
}

\begin{abstract}
Energetic solutions to rate-independent processes are usually constructed via time-incremental minimization problems. In this work we show that all energetic solutions can be approximated by such incremental problems if we allow for approximate minimizers, where the error in minimization has to be of the order of the time step. Moreover, we study sequences of problems where the energy functionals have a $\Gamma$-limit.
\end{abstract}

Mathematics Subject Classification (2000). 49J40, 49S05, 65J15, 74C05, 74H15.

Keywords. Rate-independent processes, energetic solutions, approximate incremental problems, gamma convergence.

\section{Introduction}

The concept of energetic solutions to rate-independent processes was introduced in $[22,23]$ and further developed for example in $[12,16]$; a recent survey is [19]. They allow for a mathematical treatment of a variety of evolution problems in the material sciences, for example in elastoplasticity $[5,14,28]$, phase transitions in shape-memory alloys $[1,2,15,25]$ and crack formation in brittle materials $[9,11]$. Optimal control problems based on energetic solutions are investigated in [29].

Recently, in [24] sequences of such problems have been studied in the framework of $\Gamma$-convergence and conditions were derived that guarantee that solutions of the problems in the sequence admit a limit point solving the limit problem. Here we go the opposite direction and show that every solution to the limit problem originates from time-discrete solutions to the approximate problems. This shows that the limit problem can be used effectively in the study of sequences of rate-independent problems. Roughly speaking, the theory in [24] states that the solution set is upper semi-continuous in the $\Gamma$-limit, whereas here we study the lower semi-continuity.

Research partially supported by Deutsche Forschungsgemeinschaft via the MATHEON project C18. 
We now describe the general framework in order to introduce the main ideas. Precise technical assumptions are postponed until Sections 2 and 4 .

Let the state space $\mathcal{Q}$ of the system be the product of two Hausdorff topological spaces $\mathcal{F}$ and $\mathcal{Z}$. As we will deal with sequences rather than with general topology tools, all topological notions are to be understood in a sequential sense. For example, compactness always means sequential compactness. Here $\mathcal{F}$ corresponds to the elastic (or, more generally, non-dissipative) and $\mathcal{Z}$ to the internal (or dissipative) variables. This splitting is typical in continuum mechanics with dissipation, see $[10,13,14,32]$. The system itself is modeled by two functionals: an energy-storage functional $\mathcal{E}:[0, T] \times \mathcal{Q} \rightarrow \mathbb{R}_{\infty}:=\mathbb{R} \cup\{+\infty\}$ and a dissipation distance $\mathcal{D}: \mathcal{Z} \times \mathcal{Z} \rightarrow[0, \infty]$. The triple $(\mathcal{Q}, \mathcal{E}, \mathcal{D})$ is called an energetic rate-independent system.

The energy $\mathcal{E}$ models the elastic or non-dissipative part of the problem and depends on the process time via a time-dependent loading. The value $\mathcal{D}\left(z_{0}, z_{1}\right)$ denotes the minimal dissipated energy when the state is changed from $z_{0} \in \mathcal{Z}$ to $z_{1} \in \mathcal{Z}$. Because of this physical interpretation, we require the triangle inequality and the positivity $\mathcal{D}\left(z_{1}, z_{2}\right)=0$ if and only if $z_{1}=z_{2}$. However, we do not require $\mathcal{D}$ to be symmetric as the physical dissipation might not have this property, e.g. in elastoplasticity [14], in crack formation in brittle materials [9,11], or in damage $[3,10]$. Although $\mathcal{D}$ acts only on the dissipative part $\mathcal{Z}$ of the underlying state space $\mathcal{Q}$, for $q_{1}=\left(\phi_{1}, z_{1}\right)$ and $q_{2}=\left(\phi_{2}, z_{2}\right)$ we also write $\mathcal{D}\left(q_{1}, q_{2}\right)$ when in fact we mean $\mathcal{D}\left(z_{1}, z_{2}\right)$.

For a process $z:[0, T] \rightarrow \mathcal{Z}$ (only in the dissipation part of the state space) and $s, t \in[0, T]$, define the total dissipation $\operatorname{Diss}_{\mathcal{D}}(z ;[s, t])$ of $z$ in the subinterval $[s, t]$ to be the total variation of $z$ with respect to the quasimetric $\mathcal{D}$, i.e.

$\operatorname{Diss}_{\mathcal{D}}(z ;[s, t]):=\sup \left\{\sum_{j=1}^{N} \mathcal{D}\left(z\left(\tau_{j-1}\right), z\left(\tau_{j}\right)\right): s=\tau_{0}<\cdots<\tau_{N}=t, N \in \mathbb{N}\right\}$.

Again, for a process $q:[0, T] \rightarrow \mathcal{F} \times \mathcal{Z}=\mathcal{Q}$ with $q(t)=(\phi(t), z(t))$, we also write $\operatorname{Diss}_{\mathcal{D}}(q ;[s, t])$ when we really mean $\operatorname{Diss}_{\mathcal{D}}(z ;[s, t])$.

An energetic solution to the evolution system associated with $\mathcal{E}$ and $\mathcal{D}$ is a process $q:[0, T] \rightarrow \mathcal{Q}$ that satisfies the stability condition (S) and the energy balance $(\mathrm{E})$ for all $t \in[0, T]$ :

$$
\begin{aligned}
& \text { (i) } \mathcal{E}(t, q(t)) \leq \mathcal{E}(t, \hat{q})+\mathcal{D}(q(t), \hat{q}) \quad \text { for all } \quad \hat{q} \in \mathcal{Q} \\
& \text { (ii) } \mathcal{E}(t, q(t))+\operatorname{Diss}_{\mathcal{D}}(q ;[0, t])=\mathcal{E}(0, q(0))+\int_{0}^{t} \partial_{t} \mathcal{E}(\tau, q(\tau)) \mathrm{d} \tau
\end{aligned}
$$

In this case, we also say that $q$ is a solution of the energetic rate-independent system $(\mathcal{Q}, \mathcal{E}, \mathcal{D})$.

The so-called stable sets

$$
\mathcal{S}(t):=\{q \in \mathcal{Q}: \mathcal{E}(t, q)<\infty \text { and } \mathcal{E}(t, q) \leq \mathcal{E}(t, \hat{q})+\mathcal{D}(q, \hat{q}) \text { for all } \hat{q} \in \mathcal{Q}\}
$$


play a vital role in the theory and allow condition $(\mathrm{S})$ to be rephrased into

$$
q(t) \in \mathcal{S}(t) \text { for all } t \in[0, T] .
$$

Additionally to $(\mathrm{S}) \&(\mathrm{E})$, we prescribe a stable initial value $q(0)=q_{0} \in \mathcal{S}(0)$.

In the case that $\mathcal{Q}$ is a Banach space, $\mathcal{E}$ is convex and differentiable, and $\mathcal{D}$ is given through $\mathcal{D}\left(z_{1}, z_{2}\right)=\mathcal{R}\left(z_{2}-z_{1}\right)$ with a convex, 1-homogeneous potential $\mathcal{R}: \mathcal{Z} \rightarrow[0, \infty]$, this notion is equivalent to the doubly-nonlinear differential inclusion (cf. [6])

$$
0 \in \partial \mathcal{R}(\dot{q}(t))+\mathrm{D} \mathcal{E}(t, q(t)) \text { in } \mathcal{Q}^{*}
$$

and the variational inequality

$$
\langle\mathrm{D} \mathcal{E}(t, q(t)), v-\dot{q}(t)\rangle+\mathcal{R}(v)-\mathcal{R}(\dot{q}(t)) \geq 0 \quad \text { for all } \quad v \in \mathcal{Q},
$$

cf. $[19,23]$. In this setting, the notion of rate-independence manifests itself through the 1-homogeneity of $\mathcal{R}$. In contrast to (SF) and (VI), however, the energetic formulation $(\mathrm{S}) \&(\mathrm{E})$ is derivative-free and no linear structure of $\mathcal{Q}$ needs to be assumed. This allows for the treatment of more general problems in continuum mechanics, cf. Section 7 of [19] for a survey.

In the main existence proof of the theory one constructs approximate solutions using a time-incremental problem. For this, let $\Pi=\left(0, t_{1}, \ldots, t_{N-1}, T\right)$ be a partition of the interval $[0, T]$ and consider:

$$
\left\{\begin{array}{l}
\text { For } j=1, \ldots, N, \text { inductively find } q_{j} \in \mathcal{Q} \text { such that } \\
q_{j} \in \operatorname{Argmin}\left\{\mathcal{E}\left(t_{j}, \hat{q}\right)+\mathcal{D}\left(q_{j-1}, \hat{q}\right): \hat{q} \in \mathcal{Q}\right\} .
\end{array}\right.
$$

The existence result is then obtained by constructing limits of the discrete solutions $\left(q_{j}^{n}\right)_{j=0, \ldots, N_{n}}$ of $\left(\mathrm{IP}^{\Pi_{n}}\right)$, where $\Pi_{n}=\left(t_{1}^{n}, t_{1}^{n}, \ldots, t_{N_{n}}^{n}\right)$ is a sequence of partitions whose fineness

$$
\left\|\Pi_{n}\right\|:=\max _{k=1, \ldots, N_{n}}\left(t_{k}^{n}-t_{k-1}^{n}\right) .
$$

tends to 0 as $n \rightarrow \infty$. Then, with the help of a generalized Helly's selection principle, we obtain a subsequence converging pointwise to a limit and this limit is shown to be a solution, cf. [19] for a full exposition. One can now pose the question whether every solution of $(\mathrm{S})$ \& $(\mathrm{E})$ can be obtained in such a way. This, unfortunately, is not true in general (see Counterexample 2.4). For many purposes, however, it suffices to show that we can find a solution to an $\varepsilon$-approximate incremental problem for $\varepsilon>0$ and suitable partitions $\Pi=\left(0, t_{1}, \ldots, t_{N-1}, T\right)$ of $[0, T]:$

$$
\left\{\begin{array}{l}
\text { For } j=1, \ldots, N, \text { inductively find } q_{j} \in \mathcal{Q} \text { such that } \\
q_{j} \in \operatorname{Argmin}_{\varepsilon}\left\{\mathcal{E}\left(t_{j}, \hat{q}\right)+\mathcal{D}\left(q_{j-1}, \hat{q}\right): \hat{q} \in \mathcal{Q}\right\}
\end{array}\right.
$$

where we employed the set of $\varepsilon$-minimizers, $\varepsilon \geq 0$, which, for a functional $F$ : $\mathcal{X} \rightarrow \mathbb{R}_{\infty}$, is defined as

$$
\operatorname{Argmin}_{\varepsilon}(F)=\operatorname{Argmin}_{\varepsilon}\{F(x): x \in \mathcal{X}\}:=\left\{x \in \mathcal{X}: F(x) \leq \inf _{\mathcal{X}} F+\varepsilon\right\} .
$$


In Section 2, we will show the reverse approximability of all solutions to (S) \& (E) by discrete solutions to $\left(\operatorname{AIP}_{\varepsilon}^{\Pi}\right)$ with $\varepsilon=c_{\mathrm{R}}\|\Pi\|$, where $c_{\mathrm{R}}$ is the reverse approximation constant.

Afterwards, we investigate reverse approximability for sequences of problems. These are given through sequences of energy functionals $\left(\mathcal{E}_{k}\right)_{k}$ and dissipation distances $\left(\mathcal{D}_{k}\right)_{k}$. For the $k$ th problem, $k \in \mathbb{N}_{\infty}=\mathbb{N} \cup\{\infty\}$, we denote by $\left(\mathrm{S}_{k}\right)$ and $\left(\mathrm{E}_{k}\right)$ the solution conditions corresponding to $(\mathrm{S})$ and $(\mathrm{E})$, respectively. To treat such sequences of problems, approximate incremental problems for sequences are employed, cf. [24]: Let $\Pi=\left(0, t_{1}, \ldots, t_{N-1}, T\right)$ be a partition of the interval $[0, T]$, let $\varepsilon>0$, and consider:

$$
\left\{\begin{array}{l}
\text { For } j=1, \ldots, N \text {, inductively find } q_{j} \in \mathcal{Q} \text { such that } \\
q_{j} \in \operatorname{Argmin}_{\varepsilon}\left\{\mathcal{E}_{k}\left(t_{j}, \hat{q}\right)+\mathcal{D}_{k}\left(q_{j-1}, \hat{q}\right): \hat{q} \in \mathcal{Q}\right\} .
\end{array}\right.
$$

Obviously, this problem always has a solution. In Section 4 of [24] it is shown that subsequences of solutions to $\left(\mathrm{AIP}_{k, \varepsilon}^{\Pi}\right)$ converge (in a certain sense) to a solution of $\left(\mathrm{S}_{\infty}\right) \&\left(\mathrm{E}_{\infty}\right)$. As for single problems, one can be interested in the reverse question: To a given solution to $\left(\mathrm{S}_{\infty}\right) \&\left(\mathrm{E}_{\infty}\right)$ can one find solutions to the corresponding approximate incremental problems $\left(\mathrm{AIP}_{k, \varepsilon}^{\Pi}\right)$ ? This question is answered positively in Section 4.

In other words, [24] shows that the limes superior (here in the topological or Painlevé-Kuratowski sense with respect to pointwise convergence in $\mathcal{Q}$ ) of (interpolants of ) the time-discrete solutions for the $k$ th functionals $\mathcal{E}_{k}$ and $\mathcal{D}_{k}$ on increasingly finer partitions is contained in the set of time-continuous solutions to the limit problem associated with $\mathcal{E}_{\infty}$ and $\mathcal{D}_{\infty}$. We here show that also a reverse inclusion holds (with slightly different choices of the partition fineness).

In Section 4 we also show that one cannot expect to find time-continuous solutions to $\left(\mathrm{S}_{k}\right) \&\left(\mathrm{E}_{k}\right)$, which approximate a solution to $\left(\mathrm{S}_{\infty}\right) \&\left(\mathrm{E}_{\infty}\right)$.

Section 5 provides a more quantitative approach by assuming that everything is defined in Banach spaces and that the $\Gamma$-convergence is more explicit. In particular, we discuss a kind of backward error analysis for space-time discretizations for a phase transformation model that could be easily be generalized to elastoplasticity as discussed in [14]. Finally, Section 6 discusses the relations to regularization and relaxation.

The forthcoming work [30] will apply the results presented in this paper to approximation schemes for optimal control problems involving rate-independent systems.

\section{Approximation for single problems}

In this section, the approximability of solutions to $(\mathrm{S}) \&(\mathrm{E})$ by discrete solutions to $\left(\mathrm{AIP}_{\varepsilon}^{\Pi}\right)$ is investigated. 
On $\mathcal{E}$ and $\mathcal{D}$ consider the following standard assumptions, cf. [19] for an explanation of their physical relevance:

Control of the power $\partial_{t} \mathcal{E}$ :

There exist $c_{0}^{E} \in \mathbb{R}, c_{1}^{E}>0$ such that:

If $q \in \mathcal{Q}$ satisfies $\mathcal{E}(s, q)<\infty$ for some $s \in[0, T]$, then

(i) $\mathcal{E}(., q) \in \mathrm{C}^{1}([0, T])$ and

(ii) $\left|\partial_{t} \mathcal{E}(t, q)\right| \leq c_{1}^{E}\left(\mathcal{E}(t, q)+c_{0}^{E}\right) \quad$ for all $t \in[0, T]$.

Quasimetric:

For all $z_{1}, z_{2}, z_{3} \in \mathcal{Z}$ :

(i) $\mathcal{D}\left(z_{1}, z_{2}\right)=0$ if and only if $z_{1}=z_{2}$ (positivity) and

(ii) $\mathcal{D}\left(z_{1}, z_{3}\right) \leq \mathcal{D}\left(z_{1}, z_{2}\right)+\mathcal{D}\left(z_{2}, z_{3}\right)$ (triangle inequality).

By the Gronwall lemma, (2.E) immediately implies

$$
\mathcal{E}(t, q)+c_{0}^{E} \leq\left(\mathcal{E}(s, q)+c_{0}^{E}\right) \mathrm{e}^{c_{1}^{E}|t-s|} \quad \text { for all } t, s \in[0, T], q \in \mathcal{Q} .
$$

Applying this estimate on (2.E), we get

$$
\left|\partial_{t} \mathcal{E}(t, q)\right| \leq c_{1}^{E}\left(\mathcal{E}(s, q)+c_{0}^{E}\right) \mathrm{e}^{c_{1}^{E}|t-s|} \quad \text { for all } t, s \in[0, T], \quad q \in \mathcal{Q} .
$$

Hence, for an energetic solution $q:[0, T] \rightarrow \mathcal{Q}$ and for all $t \in[0, T]$, we have the a-priori estimates

$$
\begin{aligned}
\mathcal{E}(t, q(t))+c_{0}^{E} & \leq\left(\mathcal{E}(0, q(0))+c_{0}^{E}\right) \mathrm{e}^{c_{1}^{E} t}, \\
\left|\partial_{t} \mathcal{E}(t, q(t))\right| & \leq c_{1}^{E}\left(\mathcal{E}(0, q(0))+c_{0}^{E}\right) \mathrm{e}^{c_{1}^{E} t}, \\
\operatorname{Diss}_{\mathcal{D}}(q ;[0, t]) & \leq\left(\mathcal{E}(0, q(0))+c_{0}^{E}\right) \mathrm{e}^{c_{1}^{E} t},
\end{aligned}
$$

cf. Section 3.1 in [19].

Throughout this section, we silently assume (2.E) and (2.D) to hold. Note that while here we only require these two conditions, for the existence of a solution all results known so far need additional assumptions, cf. [19,23].

We commence with a lemma which allows us to estimate the energy of approximate minimizers.

Lemma 2.1. Let $q:[0, T] \rightarrow \mathcal{Q}$ be a solution of $(\mathrm{S}) \mathscr{E}(\mathrm{E})$ with initial value $q_{0}=q(0) \in \mathcal{S}(0)$ and let $\Pi=\left(0, t_{1}, \ldots, t_{N-1}, T\right)$ be a partition of the interval $[0, T]$. Let

$$
q_{j}:=q\left(t_{j}\right) \quad \text { for } \quad j=1, \ldots, N-1 .
$$

Then, for all $\delta \geq 0$ there exists $M=M\left(q_{0}, \delta\right) \in \mathbb{R}$ such that for all $j=1, \ldots, N$ and

$$
q_{j}^{*} \in \operatorname{Argmin}_{\delta}\left\{\mathcal{E}\left(t_{j}, \hat{q}\right)+\mathcal{D}\left(q_{j-1}, \hat{q}\right): \hat{q} \in \mathcal{Q}\right\},
$$

it holds that $\mathcal{E}\left(s, q_{j}^{*}\right) \leq M$ for all $s \in[0, T]$. Further, $M\left(q_{0}, \delta\right)$ can be chosen increasing in $\delta$. 
Proof. First, we use the $\delta$-minimality of $q_{j}^{*}$ to derive

$$
\begin{aligned}
\mathcal{E}\left(t_{j}, q_{j}^{*}\right)+\mathcal{D}\left(q_{j-1}, q_{j}^{*}\right) & \leq \mathcal{E}\left(t_{j}, q_{j-1}\right)+\mathcal{D}\left(q_{j-1}, q_{j-1}\right)+\delta \\
& =\mathcal{E}\left(t_{j-1}, q_{j-1}\right)+\int_{t_{j-1}}^{t_{j}} \partial_{t} \mathcal{E}\left(\tau, q_{j-1}\right) \mathrm{d} \tau+\delta,
\end{aligned}
$$

where we exploited $\mathcal{D}\left(q_{j-1}, q_{j-1}\right)=0$. We can now use the growth estimate $(2.2)$ to deduce

$$
\begin{aligned}
\int_{t_{j-1}}^{t_{j}} \partial_{t} \mathcal{E}\left(\tau, q_{j-1}\right) \mathrm{d} \tau & \leq \int_{t_{j-1}}^{t_{j}} c_{1}^{E}\left(\mathcal{E}\left(t_{j-1}, q_{j-1}\right)+c_{0}^{E}\right) \mathrm{e}^{c_{1}^{E}\left(\tau-t_{j-1}\right)} \mathrm{d} \tau \\
& =\left(\mathcal{E}\left(t_{j-1}, q_{j-1}\right)+c_{0}^{E}\right)\left(\mathrm{e}^{c_{1}^{E}\left(t_{j}-t_{j-1}\right)}-1\right) .
\end{aligned}
$$

The a-priori bound (2.3) on the energy of the continuous solution provides the necessary information to estimate the term $\mathcal{E}\left(t_{j-1}, q_{j-1}\right)$. Indeed,

$$
\mathcal{E}\left(t_{j-1}, q_{j-1}\right)+c_{0}^{E} \leq\left(\mathcal{E}\left(0, q_{0}\right)+c_{0}^{E}\right) \mathrm{e}^{c_{1}^{E} t_{j-1}} \leq\left(\mathcal{E}\left(0, q_{0}\right)+c_{0}^{E}\right) \mathrm{e}^{c_{1}^{E} T}=: L
$$

where $L=L\left(q_{0}, c_{0}^{E}, c_{1}^{E}\right)$ only depends on $q_{0}, c_{0}^{E}$, and $c_{1}^{E}$. We combine this with the previous estimates $(2.6),(2.7)$ to get

$$
\begin{aligned}
\mathcal{E}\left(t_{j}, q_{j}^{*}\right) & \leq \mathcal{E}\left(t_{j}, q_{j}^{*}\right)+\mathcal{D}\left(q_{j-1}, q_{j}^{*}\right) \\
& \leq \mathcal{E}\left(t_{j-1}, q_{j-1}\right)+\left(\mathcal{E}\left(t_{j-1}, q_{j-1}\right)+c_{0}^{E}\right)\left(\mathrm{e}^{c_{1}^{E}\left(t_{j}-t_{j-1}\right)}-1\right)+\delta \\
& \leq L+L\left(\mathrm{e}^{c_{1}^{E} T}-1\right)+\delta=: L_{1}=L_{1}\left(q_{0}, \delta\right) .
\end{aligned}
$$

Using (2.1) with $s=t_{j}$, the result follows with $M=\left(L_{1}\left(q_{0}, \delta\right)+c_{0}^{E}\right) \mathrm{e}^{c_{1}^{E} T}-c_{0}^{E}$. The monotonicity claim is clear.

We are now in a position to prove that every solution of $(\mathrm{S}) \&(\mathrm{E})$ gives rise to a solution of $\left(\mathrm{AIP}_{\varepsilon}^{\Pi}\right)$.

Theorem 2.2. Let $q:[0, T] \rightarrow \mathcal{Q}$ be a solution of $(\mathrm{S})$ \& $(\mathrm{E})$ with initial value $q_{0}=q(0) \in \mathcal{S}(0)$. Then, there exists a constant $c_{\mathrm{R}}=c_{\mathrm{R}}\left(q_{0}\right)>0$ such that for any partition $\Pi=\left(0, t_{1}, \ldots, t_{N-1}, T\right)$ of the interval $[0, T]$, the values $q_{j}:=q\left(t_{j}\right)$, $j=1, \ldots, N$, solve $\left(\mathrm{AIP}_{\varepsilon}^{\Pi}\right)$ with $\varepsilon=c_{\mathrm{R}}\|\Pi\|$, i.e.

$$
q_{j} \in \operatorname{Argmin}_{c_{\mathrm{R}}\|\Pi\|}\left\{\mathcal{E}\left(t_{j}, \hat{q}\right)+\mathcal{D}\left(q_{j-1}, \hat{q}\right): \hat{q} \in \mathcal{Q}\right\} \quad \text { for } \quad j=1, \ldots, N .
$$

The quantity $c_{\mathrm{R}}=c_{\mathrm{R}}\left(q_{0}\right)$ is called the reverse approximation constant of the problem.

Proof. The energy balance (E) implies

$$
\mathcal{E}\left(t_{j}, q_{j}\right)+\operatorname{Diss}_{\mathcal{D}}\left(q ;\left[t_{j-1}, t_{j}\right]\right)=\mathcal{E}\left(t_{j-1}, q_{j-1}\right)+\int_{t_{j-1}}^{t_{j}} \partial_{t} \mathcal{E}(\tau, q(\tau)) \mathrm{d} \tau .
$$

The stability $q_{j-1} \in \mathcal{S}\left(t_{j-1}\right)$ gives $\mathcal{E}\left(t_{j-1}, q_{j-1}\right) \leq \mathcal{E}\left(t_{j-1}, \hat{q}\right)+\mathcal{D}\left(q_{j-1}, \hat{q}\right)$ for all $\hat{q} \in \mathcal{Q}$. Together with $\mathcal{D}\left(q_{j-1}, q_{j}\right) \leq \operatorname{Diss}_{\mathcal{D}}\left(q ;\left[t_{j-1}, t_{j}\right]\right)$ this gives

$$
\mathcal{E}\left(t_{j}, q_{j}\right)+\mathcal{D}\left(q_{j-1}, q_{j}\right) \leq \mathcal{E}\left(t_{j-1}, \hat{q}\right)+\mathcal{D}\left(q_{j-1}, \hat{q}\right)+\int_{t_{j-1}}^{t_{j}} \partial_{t} \mathcal{E}(\tau, q(\tau)) \mathrm{d} \tau,
$$


and we continue by estimating the integral term using the growth estimate (2.4) to find

$$
\begin{aligned}
\int_{t_{j-1}}^{t_{j}} \partial_{t} \mathcal{E}(\tau, q(\tau)) \mathrm{d} \tau & \leq \int_{t_{j-1}}^{t_{j}} c_{1}^{E}\left(\mathcal{E}\left(0, q_{0}\right)+c_{0}^{E}\right) \mathrm{e}^{c_{1}^{E} \tau} \mathrm{d} \tau \\
& \leq c_{1}^{E}\left(\mathcal{E}\left(0, q_{0}\right)+c_{0}^{E}\right) \mathrm{e}^{c_{1}^{E} T}\left(t_{j}-t_{j-1}\right) .
\end{aligned}
$$

Assuming $\mathcal{E}\left(t_{j-1}, \hat{q}\right)<\infty$, the quantity $\mathcal{E}\left(t_{j-1}, \hat{q}\right)$ can be estimated using $(2.2)$ :

$$
\begin{aligned}
\mathcal{E}\left(t_{j-1}, \hat{q}\right) & =\mathcal{E}\left(t_{j}, \hat{q}\right)-\int_{t_{j-1}}^{t_{j}} \partial_{t} \mathcal{E}(\tau, \hat{q}) \mathrm{d} \tau \\
& \leq \mathcal{E}\left(t_{j}, \hat{q}\right)+\int_{t_{j-1}}^{t_{j}} c_{1}^{E}\left(\mathcal{E}(0, \hat{q})+c_{0}^{E}\right) \mathrm{e}^{c_{1}^{E} \tau} \mathrm{d} \tau
\end{aligned}
$$

Now choose $\hat{q}:=q_{j}^{*}$ with $q_{j}^{*} \in \operatorname{Argmin}_{\delta}\left\{\mathcal{E}\left(t_{j}, \hat{q}\right)+\mathcal{D}\left(q_{j-1}, \hat{q}\right): \hat{q} \in \mathcal{Q}\right\}$ for some $0<\delta \leq \min \{1,\|\Pi\|\}$. Such a $q_{j}^{*}$ always exists, and $\mathcal{E}\left(s, q_{j}^{*}\right)<\infty$ for all $s \in[0, T]$. By Lemma 2.1 , we can bound $\mathcal{E}\left(0, q_{j}^{*}\right)$ in $(2.10)$ by a constant $M=M\left(q_{0}, \delta\right) \leq$ $M\left(q_{0}, 1\right)=: M_{1}$ (note the monotonicity of $M$ in $\delta$ ), which does not depend on $\Pi$ (or any other quantities except $q_{0}$ ). This gives

$$
\mathcal{E}\left(t_{j-1}, q_{j}^{*}\right) \leq \mathcal{E}\left(t_{j}, q_{j}^{*}\right)+c_{1}^{E}\left(M_{1}+c_{0}^{E}\right) \mathrm{e}^{c_{1}^{E} T}\left(t_{j}-t_{j-1}\right) .
$$

Plugging (2.9) and (2.11) into (2.8), we see

$$
\begin{aligned}
\mathcal{E}\left(t_{j}, q_{j}\right)+\mathcal{D}\left(q_{j-1}, q_{j}\right) \leq & \mathcal{E}\left(t_{j}, q_{j}^{*}\right)+\mathcal{D}\left(q_{j-1}, q_{j}^{*}\right) \\
& +c_{1}^{E}\left(\mathcal{E}\left(0, q_{0}\right)+M_{1}+2 c_{0}^{E}\right) \mathrm{e}^{c_{1}^{E} T}\|\Pi\| \\
\leq & \inf \left\{\mathcal{E}\left(t_{j}, \hat{q}\right)+\mathcal{D}\left(q_{j-1}, \hat{q}\right): \hat{q} \in \mathcal{Q}\right\}+\delta+\tilde{c}\|\Pi\|, \\
\leq & \inf \left\{\mathcal{E}\left(t_{j}, \hat{q}\right)+\mathcal{D}\left(q_{j-1}, \hat{q}\right): \hat{q} \in \mathcal{Q}\right\}+c_{\mathrm{R}}\|\Pi\|
\end{aligned}
$$

where we have set $\tilde{c}:=c_{1}^{E}\left(\mathcal{E}\left(0, q_{0}\right)+M_{1}+2 c_{0}^{E}\right) \mathrm{e}^{c_{1}^{E} T}$ and $c_{\mathrm{R}}:=1+\tilde{c}$. As $M_{1}$ only depends on $q_{0}$, so does $c_{\mathrm{R}}$, and the proof is complete.

Remark 2.3. The proof also shows that one may replace $\|\Pi\|$ by $\Delta t_{j}:=t_{j}-t_{j-1}$ in the expression for $q_{j}$.

We close this section with an example showing that one cannot expect approximability by $\left(\mathrm{IP}^{\Pi}\right)$ instead of $\left(\mathrm{AIP}_{\varepsilon}^{\Pi}\right)$.

Counterexample 2.4. On the space $\mathcal{Q}=[-1,1]$ and in the time interval $[0, T]=$ $[0,1]$, we consider the potential

$$
\mathcal{E}(t, q):=(1+q)(1-q)+\frac{t}{2} q
$$

together with the dissipation distance $\mathcal{D}(u, v):=|v-u|$. It is easily seen that this potential fulfills all the requirements of the theory on the compact interval $[-1,1]$. The functional $\mathcal{E}(t,$.$) has precisely two (strict) local minima, one at -1$ and the other at +1 . There, $\mathcal{E}$ takes the values $\mathcal{E}(t,-1)=-t / 2$ and $\mathcal{E}(t,+1)=t / 2$, respectively. The graph of $\mathcal{E}(t,$.$) lies above the supporting hyperplane through the$ 
points $(-1, \mathcal{E}(t,-1))=(-1,-t / 2)$ and $(+1, \mathcal{E}(t,+1))=(+1, t / 2)$; this hyperplane is represented by the linear map $q \mapsto t q / 2$.

At $t=0$, the set of stable states $\mathcal{S}(0)$ contains 0 , because

$$
\mathcal{E}(0, q)+\mathcal{D}(0, q)=1-q^{2}+|q| \geq 1=\mathcal{E}(0,0),
$$

since $q^{2} \leq|q|$ in $[-1,1]$. The following two processes are both solutions to the energetic formulation $(\mathrm{S}) \&(\mathrm{E})$ with initial value $q_{0}=0 \in \mathcal{S}(0)$ :

$$
q_{-}(t):=\left\{\begin{array}{l}
0 \quad \text { if } \quad t=0, \\
-1 \quad \text { if } t \in(0,1]
\end{array} \quad \text { and } \quad q_{+}(t):=\left\{\begin{array}{l}
0 \text { if } t=0 \\
+1 \text { if } t \in(0,1]
\end{array}\right.\right.
$$

We have $-1,+1 \in \mathcal{S}(t)$ for all $t \in[0,1]$. For -1 this is clear since -1 is the global minimum for all $t \geq 0$. For +1 , the hyperplane $q \mapsto t q / 2$ supports the graph of $\mathcal{E}(t,$.$) . This hyperplane has at most slope 1 / 2$; therefore, if we add the linear map $q \mapsto \mathcal{D}(+1, q)=1-q$ ( since $q \in[-1,1])$ to the graph, we still have a hyperplane with negative slope $-1 / 2$ supporting $\mathcal{E}(t,)+.\mathcal{D}(+1,$.$) and going through$ $(+1, \mathcal{E}(t,+1))$. Hence, also $+1 \in \mathcal{S}(t)$. We have thus established the validity of $(\mathrm{S})$ for $q_{-}$and $q_{+}$. Further, for $t \in(0,1]$, the energy balance (E) holds as well:

$$
\begin{aligned}
\mathcal{E}\left(t, q_{ \pm}(t)\right)+\operatorname{Diss}_{\mathcal{D}}\left(q_{ \pm} ;[0, t]\right) & =\mathcal{E}(t, \pm 1)+\mathcal{D}(0, \pm 1)=\frac{ \pm t}{2}+1 \\
& =\mathcal{E}\left(0, q_{0}\right)+\int_{0}^{t} \partial_{t} \mathcal{E}\left(\tau, q_{ \pm}(\tau)\right) \mathrm{d} \tau
\end{aligned}
$$

since $\partial_{t} \mathcal{E}\left(\tau, q_{ \pm}(\tau)\right)= \pm 1 / 2$ for almost all $\tau \in[0, T]$. For $t=0$, the energy balance is trivial.

While both $q_{-}$and $q_{+}$solve $(\mathrm{S})$ \& $(\mathrm{E})$, the incremental problem $\left(\mathrm{IP}^{\Pi}\right)$ will always select $q_{-}$: In the first step, at time $t_{1}>0$, we seek the global minimizer of $\mathcal{E}\left(t_{1},.\right)+\mathcal{D}(0,$.$) . But this global minimizer clearly is -1$. So, the discrete solution will jump to $q_{1}=-1$ and, because $-1 \in \mathcal{S}(t)$ for all $t$, stay there forever. Passing to the limit, we get the solution process $q_{-}$from above. The other solution $q_{+}$, however, is not selected. This shows that not all solutions of $(S) \&(E)$ correspond to discrete solutions if we only allow strict minimizers in the incremental problem.

Note that $\mathcal{E}(t,$.$) is the restriction of the double-well potential$

$$
(t, q) \mapsto|1-q||1+q|+\frac{t}{2} q
$$

to the interval $[-1,1]$. We refrained from carrying out the example on a bigger space for ease of notation only; everything works just the same for bigger intervals.

Hence, this example represents physically a phase-transition problem, where the energies of the two phases change in the course of time due to a prescribed loading $(q \mapsto t q / 2)$. Our results therefore reflect that while there might be a "preferred" solution $q_{-}$, another solution, namely $q_{+}$, can also occur if we allow for small (in fact, arbitrarily small) perturbations. This is nothing else but the instability of rate-independent evolution processes and seems to be well in line with physical intuition. 
Remark 2.5. The last counterexample also shows that the error order $\varepsilon=\mathrm{O}(\|\Pi\|)$ is optimal: If the solution $q_{+}$is to be selected, the discrete solution must jump from 0 to +1 at time $t_{1}$. The difference between $\mathcal{E}\left(t_{1},-1\right)$ and $\mathcal{E}\left(t_{1},+1\right)$ is $t_{1}$, hence the error in the minimization of $\mathcal{E}\left(t_{1},.\right)+\mathcal{D}(0,$.$) is t_{1}=\mathrm{O}(\|\Pi\|)$ and nothing better than linear order can be achieved.

In summary, the results of the this section suggest that $\left(\mathrm{AIP}_{\varepsilon}^{\Pi}\right)$ is better suited than $\left(\mathrm{IP}^{\Pi}\right)$ as a time discretization of $(\mathrm{S}) \&(\mathrm{E})$. This holds especially in numerical applications, cf. Section 5.

\section{Sets of approximate minimizers and $\Gamma$-convergence}

In this section, we provide some auxiliary results on $\varepsilon$-minimizers of $\Gamma$-converging functionals. This preliminary considerations will be needed in the following section. The result is stated in a little more generality than what is needed subsequently in order to clarify ideas and avoid too much notational clutter.

In the following, let $\mathcal{X}$ be a Hausdorff space and, just like in Section 2, all topological notions are to be understood in a sequential sense. The functional $F_{\infty}: \mathcal{X} \rightarrow \mathbb{R}_{\infty}$ is called the (sequential) $\Gamma$-limit of the sequence $\left(F_{k}\right)_{k}$ of functionals $F_{k}: \mathcal{X} \rightarrow \mathbb{R}_{\infty}$, if it satisfies the following two conditions:

(i) For all $x \in \mathcal{X}$ and all $\left(x_{k}\right)_{k}$ with $x_{k} \rightarrow x$ the lim inf-inequality holds:

$$
F_{\infty}(x) \leq \liminf _{k \rightarrow \infty} F_{k}\left(x_{k}\right)
$$

(ii) For all $x \in \mathcal{X}$ there exists a recovery sequence $\left(x_{k}\right)_{k}$,

$$
\text { i.e. } x_{k} \rightarrow x \text { and } F_{\infty}(x)=\lim _{k \rightarrow \infty} F_{k}\left(x_{k}\right) \text {. }
$$

The (unique) $\Gamma$-limit of the sequence $\left(F_{k}\right)_{k}$ is denoted by $F_{\infty}=\Gamma-\lim _{k} F_{k}$.

Here, only the sequential notion of $\Gamma$-convergence is employed even though $\mathcal{X}$ might not be first countable, in which case sequential and general topological concepts differ. Still, in the calculus of variations it is often more convenient to use sequences instead of neighborhoods and nets. For $\Gamma$-convergence in general topological spaces, see [8].

We also need the following notion of uniform coercivity: A family $\left(F_{k}\right)_{k}$, $k \in \mathbb{N}$, of functionals $F_{k}: \mathcal{X} \rightarrow \mathbb{R}_{\infty}$ is called equi-mildly coercive if there exists a compact set $K \subseteq \mathcal{X}$ with

$$
\inf _{\mathcal{X}} F_{k}=\inf _{K} F_{k} \quad \text { for all } k \in \mathbb{N} \text {. }
$$

The first result of this section shows that $\varepsilon$-minimizers of $F_{\infty}$ can be approximated arbitrarily well by $(\varepsilon+\delta)$-minimizers of the $F_{k}$, where $\delta>0$.

Proposition 3.1. Let $F_{k}: \mathcal{X} \rightarrow \mathbb{R}_{\infty}, k \in \mathbb{N}$, be equi-mildly coercive functionals and assume $F_{\infty}=\Gamma-\lim _{k} F_{k}$. Then, for all $\varepsilon \geq 0$ and $x \in \operatorname{Argmin}_{\varepsilon}\left(F_{\infty}\right)$ and all recovery sequences $\left(x_{k}\right)_{k}$ for $x$ (in particular $x_{k} \rightarrow x$ ) and for every $\delta>0$ it holds that $x_{k} \in \operatorname{Argmin}_{\varepsilon+\delta}\left(F_{k}\right)$ for all $k \geq k_{0}=k_{0}(\delta)$ with $k_{0}(\delta)$ sufficiently large. 
Proof. From the convergence of infima with respect to $\Gamma$-convergence for equimildly coercive functionals $[4,8]$, we know that $\inf _{\mathcal{X}} F_{\infty}=\lim _{k \rightarrow \infty} \inf \mathcal{X}_{\mathcal{X}} F_{k}$. Hence, for $k$ sufficiently large, it holds that

$$
\left|\inf _{\mathcal{X}} F_{\infty}-\inf _{\mathcal{X}} F_{k}\right| \leq \frac{\delta}{2}
$$

Let now $\left(x_{k}\right)_{k}$ be a recovery sequence at $x$, i.e. $x_{k} \rightarrow x$ and $F_{\infty}(x)=\lim _{k \rightarrow \infty} F_{k}\left(x_{k}\right)$ (because $F_{\infty}=\Gamma$ - $\lim _{k} F_{k}$, there exists at least one such sequence). This implies, again for $k$ big enough, that

$$
\left|F_{k}\left(x_{k}\right)-F_{\infty}(x)\right| \leq \frac{\delta}{2}
$$

Combining these two estimates with $\left|F_{\infty}(x)-\inf _{\mathcal{X}} F_{\infty}\right| \leq \varepsilon$ yields

$$
\begin{aligned}
\left|F_{k}\left(x_{k}\right)-\inf _{\mathcal{X}} F_{k}\right| & \leq\left|F_{k}\left(x_{k}\right)-F_{\infty}(x)\right|+\left|F_{\infty}(x)-\inf _{\mathcal{X}} F_{\infty}\right|+\left|\inf _{\mathcal{X}} F_{\infty}-\inf _{\mathcal{X}} F_{k}\right| \\
& \leq \frac{\delta}{2}+\varepsilon+\frac{\delta}{2}=\varepsilon+\delta
\end{aligned}
$$

i.e. $x_{k} \in \operatorname{Argmin}_{\varepsilon+\delta}\left(F_{k}\right)$ for all $k$ sufficiently large.

Remark 3.2. An inspection of the proof reveals that if $\inf _{\mathcal{X}} F_{k}=\inf _{\mathcal{X}} F_{\infty}$ for all $k \in \mathbb{N}$, then we do not need the assumptions of equi-mild coerciveness as it is only needed for convergence of infima. This equality of infima is indeed easy to fulfill in the calculus of variations: We can always set $F_{k}^{\prime}:=F_{k}+c_{k}$, where $c_{k}$ is chosen precisely to ensure equality of infima. This translation does not change the minimization problem associated with $F_{k}$, in particular $\operatorname{Argmin} F_{k}=\operatorname{Argmin} F_{k}^{\prime}$.

One could hope to avoid the usage of the sequence $\left(x_{k}\right)_{k}$ and conjecture that an $\varepsilon$-minimizer of $F_{\infty}$ is also an $n \varepsilon$-minimizer of $F_{k}$ for some $n \in \mathbb{N}$ and for $k$ sufficiently large. Even if all the $F_{k}$ are lower semicontinuous, however, this is not the case as shown by the following counterexample.

Counterexample 3.3. Let $\mathcal{X}=[-1,1]$ and for $k \in \mathbb{N}$ define

$$
F_{k}(x):=\left\{\begin{array}{l}
-1 \text { if } x=1 / k, \\
0 \text { otherwise, }
\end{array} \quad \text { and } \quad F_{\infty}(x):=\left\{\begin{array}{l}
-1 \text { if } x=0, \\
0 \text { otherwise }
\end{array}\right.\right.
$$

Clearly, $F_{\infty}=\Gamma-\lim _{k} F_{k}$ and all $F_{k}, F_{\infty}$ are lower semicontinuous. However, for any $\varepsilon \in[0,1)$, the only $\varepsilon$-minimizer is $x=0$, but $x=0$ is no $(\varepsilon+\delta)$-minimizer of any $F_{k}$ as long as $\varepsilon+\delta<1$.

The next counterexample shows that in Proposition 3.1 we cannot replace the $\Gamma$-limit by the (sequential) $\Gamma$-limes inferior $\Gamma$-lim inf ${ }_{k} F_{k}$ of the sequence $\left(F_{k}\right)_{k}$, which is defined as the functional $F_{*}: \mathcal{X} \rightarrow \mathbb{R}_{\infty}$ with

$$
F_{*}=\Gamma-\liminf \operatorname{in}_{k} F_{k}:=\inf \left\{\liminf _{k \rightarrow \infty} F_{k}\left(x_{k}\right): x_{k} \rightarrow x\right\} .
$$


Counterexample 3.4. Let again $\mathcal{X}=[-1,1]$ and for all $k \in \mathbb{N}$ define

$$
G_{k}(x):=\left\{\begin{array}{l}
(-1)^{k} \text { if } x=1 / k, \\
-1 / 2 \text { if } x=1, \\
0 \text { otherwise, }
\end{array} \quad \text { and } G_{*}(x):=\left\{\begin{array}{l}
-1 \text { if } x=0, \\
-1 / 2 \text { if } x=1, \\
0 \text { otherwise. }
\end{array}\right.\right.
$$

Clearly, $G_{*}=\Gamma$ - $\liminf _{k} G_{k}$, but the $G_{k}$ do not $\Gamma$-converge. In fact, for $x=0$, one would need to construct a sequence $\left(x_{k}\right)_{k}$ with $-1=G_{*}(0) \geq \lim \sup _{k \rightarrow \infty} G_{k}\left(x_{k}\right)$. But as $G_{2 l} \geq-1 / 2$, it follows that $\lim _{\sup _{k \rightarrow \infty}} G_{k}\left(x_{k}\right) \geq-1 / 2$, which leads to the contradiction $-1 \geq-1 / 2$.

While for the subsequence $\left(x_{k_{l}}\right)_{l}$ with $k_{l}=2 l+1$ and $x_{2 l+1}=1 /(2 l+1)$ we even have $x_{2 l+1} \in \operatorname{Argmin}_{\varepsilon}\left(F_{2 l+1}\right)$ (without $\delta$ ), the conclusion of Proposition 3.1 fails, because we cannot find a "whole" sequence $\left(x_{k}\right)_{k}$ with $x_{k} \in \operatorname{Argmin}_{\varepsilon+\delta}\left(F_{k}\right)$ and $x_{k} \rightarrow x$ : The only, say, $1 / 6$-minimizer of $G_{*}$ is $x_{*}=0(\varepsilon=1 / 6)$, but any $(1 / 6+1 / 6)$-minimizer $(\delta=1 / 6)$ for $G_{2 l}$ must be $x_{2 l}=1$ and hence the sequence $\left(x_{k}\right)_{k}$ cannot converge to $x_{*}=0$.

\section{Approximation for sequences of problems}

In this section we will show that, under suitable convergence assumptions on the involved functionals, solutions to $\left(\mathrm{S}_{\infty}\right) \&\left(\mathrm{E}_{\infty}\right)$ can be approximated by solutions to $\left(\mathrm{AIP}_{k, \varepsilon}^{\Pi}\right)$. Applications are given in Sections 5 and 6 .

On the state space $\mathcal{Q}=\mathcal{F} \times \mathcal{Z}$, consider a sequence $\left(\mathcal{E}_{k}\right)_{k}$ of energy-storage functionals $\mathcal{E}_{k}:[0, T] \times \mathcal{Q} \rightarrow \mathbb{R}_{\infty}$ as well as a limit energy $\mathcal{E}_{\infty}:[0, T] \times \mathcal{Q} \rightarrow \mathbb{R}_{\infty}$. We require these functionals to fulfill the following assumption, where, for brevity, we denote by $\mathbb{N}_{\infty}$ the set $\mathbb{N} \cup\{\infty\}$ :

Uniform control of the power $\partial_{t} \mathcal{E}_{k}$ :

There exist $c_{0}^{E} \in \mathbb{R}, c_{1}^{E}>0$ such that for all $k \in \mathbb{N}_{\infty}$ :

If $q \in \mathcal{Q}$ satisfies $\mathcal{E}_{k}(s, q)<\infty$ for some $s \in[0, T]$, then

(i) $\mathcal{E}_{k}(., q) \in \mathrm{C}^{1}([0, T])$ and

(ii) $\left|\partial_{t} \mathcal{E}_{k}(t, q)\right| \leq c_{1}^{E}\left(c_{0}^{E}+\mathcal{E}_{k}(t, q)\right) \quad$ for all $t \in[0, T]$.

Equi-coercivity

For all $t \in[0, T]$ and $E \in \mathbb{R}$, the set

$\bigcup_{k \in \mathbb{N}}\left\{q \in \mathcal{Q}: \mathcal{E}_{k}(t, q) \leq E\right\}$ is relatively compact.

Further, let us be given a sequence $\left(\mathcal{D}_{k}\right)_{k}$ of dissipation distances $\mathcal{D}_{k}: \mathcal{Z} \times$ $\mathcal{Z} \rightarrow[0, \infty]$ and a limit dissipation $\mathcal{D}_{\infty}: \mathcal{Z} \times \mathcal{Z} \rightarrow[0, \infty]$. We assume:

Quasimetric:

For all $k \in \mathbb{N}_{\infty}$ and $z_{1}, z_{2}, z_{3} \in \mathcal{Z}$ :

(i) $\mathcal{D}_{k}\left(z_{1}, z_{2}\right)=0$ if and only if $z_{1}=z_{2}$ (positivity) and

(ii) $\mathcal{D}_{k}\left(z_{1}, z_{3}\right) \leq \mathcal{D}_{k}\left(z_{1}, z_{2}\right)+\mathcal{D}_{k}\left(z_{2}, z_{3}\right)$ (triangle inequality). 
Of course, the Gronwall- and a-priori estimates (2.1)-(2.5) from Section 2 now hold for all $\mathcal{E}_{k}$ with $k \in \mathbb{N}_{\infty}$.

We want the functionals $\mathcal{E}_{k}$ and $\mathcal{D}_{k}$ to converge to $\mathcal{E}_{\infty}$ and $\mathcal{D}_{\infty}$, respectively, in an appropriate sense:

$\Gamma$-limit for $\mathcal{E}_{k}$ :

For all $t \in[0, T]: \mathcal{E}_{\infty}(t,)=.\Gamma-\lim _{k} \mathcal{E}_{k}(t,)$.

Continuous convergence of $\mathcal{D}_{k}$ :

For all sequences $\left(q_{k}\right)_{k},\left(\tilde{q}_{k}\right)_{k}$ with $q_{k} \rightarrow q, \tilde{q}_{k} \rightarrow \tilde{q}$ that

additionally satisfy $\sup _{k \in \mathbb{N}}\left(\mathcal{E}_{k}\left(t, q_{k}\right)+\mathcal{E}_{k}\left(t, \tilde{q}_{k}\right)\right)<\infty$

for one (hence all) $t \in[0, T]: \mathcal{D}_{k}\left(q_{k}, \tilde{q}_{k}\right) \rightarrow \mathcal{D}_{\infty}(q, \tilde{q})$.

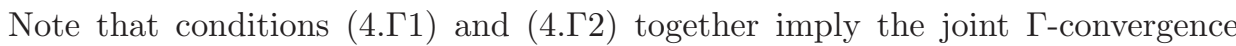
$\mathcal{E}_{\infty}(t,)+.\mathcal{D}_{\infty}(q,)=.\Gamma-\lim _{k}\left(\mathcal{E}_{k}(t,)+.\mathcal{D}_{k}(q,).\right)$ for all $t \in[0, T]$ and $q \in \mathcal{Q}$.

Example 4.1. Let $\mathcal{Q}$ be a Banach space equipped with weak sequential convergence and let $\mathcal{Q}$ be compactly embedded into another Banach space $\mathcal{Q}_{1}$. Because $\mathcal{Q} \subseteq \mathcal{Q}_{1}$, we can choose the $\mathcal{Q}_{1}-$ Norm $\|.\|_{1}$ as our dissipation distance for all $k$, i.e. $\mathcal{D}_{\infty}(u, v)=\mathcal{D}_{k}(u, v):=\mathcal{D}(u, v):=\|v-u\|_{1}$. The compact embedding then ensures the continuity of $\mathcal{D}$ and hence also the continuous convergence of $\mathcal{D}_{k}$ to $\mathcal{D}_{\infty}$.

This example shows that we can use the $\mathrm{L}^{1}(\Omega)$ norm as a weakly continuous dissipation distance in $\mathrm{H}^{1}(\Omega)$, which is a common situation in continuum mechanics [19].

After these preparations we can state the main result. It shows that solutions to $\left(\mathrm{S}_{\infty}\right) \&\left(\mathrm{E}_{\infty}\right)$ can be "reversely approximated" by solutions to $\left(\mathrm{AIP}_{k, \varepsilon}^{\Pi}\right)$.

Theorem 4.2. Let the assumptions (4.E1), (4.E2), (4.D), (4.Г1), and (4.Г2) hold. Moreover, let $q_{\infty}:[0, T] \rightarrow \mathcal{Q}$ be a solution to $\left(\mathrm{S}_{\infty}\right)$ \& $\left(\mathrm{E}_{\infty}\right)$ with initial value $q_{0}=$ $q_{\infty}(0) \in \mathcal{S}_{\infty}(0)$. Then, for all $\varepsilon>0$, for all partitions $\Pi=\left(0, t_{1}, \ldots, t_{N-1}, T\right)$ of $[0, T]$ with $\|\Pi\| \leq \varepsilon /\left(2 c_{\mathrm{R}}\right) \quad\left(c_{\mathrm{R}}=c_{\mathrm{R}}\left(q_{0}\right)>0\right.$ is the reverse approximation constant from Theorem 2.2 applied to $\mathcal{E}_{\infty}$ and $\left.\mathcal{D}_{\infty}\right)$, and for all $k$, there exist a discrete solution $q_{k}^{\Pi}:=\left(q_{0}^{k}, q_{1}^{k}, \ldots, q_{N}^{k}\right)$, defined on the partition $\Pi$, of the approximate incremental problem $\left(\mathrm{AIP}_{k, \varepsilon}^{\Pi}\right)$ associated with $\mathcal{E}_{k}$ and $\mathcal{D}_{k}$, such that $q_{j}^{k} \rightarrow q\left(t_{j}\right)$ as $k \rightarrow \infty$.

Proof. The main idea of the proof is to first construct a discrete solution to $\left(\mathrm{AIP}_{\infty, \varepsilon / 2}^{\Pi}\right)$ and then show how this discrete solution can be changed to yield a solution of $\left(\operatorname{AIP}_{k, \varepsilon}^{\Pi}\right)$ for $k$ sufficiently large. In detail, however, some further technicalities are needed.

As $\mathcal{E}_{\infty}$ and $\mathcal{D}_{\infty}$ fulfill all the prerequisites of Theorem 2.2 , for a partition $\Pi$ sufficiently fine, i.e. $\|\Pi\| \leq \varepsilon /\left(2 c_{\mathrm{R}}\right)$, we can find a discrete $\varepsilon / 2$-solution $\tilde{q}^{\Pi}=$ $\left(\tilde{q}_{0}, \tilde{q}_{1}, \ldots, \tilde{q}_{N}\right)$ for $\left(\operatorname{AIP}_{\infty, \varepsilon / 2}^{\Pi}\right)$, i.e.

$$
\tilde{q}_{j} \in \operatorname{Argmin}_{\varepsilon / 2}\left(\mathcal{E}_{\infty}\left(t_{j}, .\right)+\mathcal{D}_{\infty}\left(\tilde{q}_{j-1}, .\right)\right) \text { for } j=1, \ldots, N .
$$

Note that Theorem 2.2 uses the choice $\tilde{q}_{j}=q\left(t_{j}\right)$. 
Assumptions (4.Г1) and (4.Г2) give $\mathcal{E}_{\infty}\left(t_{j},.\right)+\mathcal{D}_{\infty}\left(\tilde{q}_{j-1},.\right)=\Gamma-\lim _{k}\left(\mathcal{E}_{k}\left(t_{j},.\right)+\right.$ $\left.\mathcal{D}_{k}\left(\tilde{q}_{j-1},.\right)\right)$. Condition (4.E2) provides the equi-mild coerciveness (3.1) (in fact, take $E:=\mathcal{E}_{\infty}\left(t_{j}, \tilde{q}_{j-1}\right)+1$ and observe that $\inf _{\mathcal{Q}}\left(\mathcal{E}_{k}\left(t_{j},.\right)+\mathcal{D}_{k}\left(\tilde{q}_{j-1},.\right)\right) \leq E$ for all $k$ large enough). Hence, using Proposition 3.1 for each $\tilde{q}_{j}$ we find recovery sequences $\left(q_{j}^{k}\right)_{k}$ with $q_{j}^{k} \rightarrow \tilde{q}_{j}$ as $k \rightarrow \infty$ and

$$
\mathcal{E}_{k}\left(t_{j}, q_{j}^{k}\right)+\mathcal{D}_{k}\left(\tilde{q}_{j-1}, q_{j}^{k}\right) \leq \inf _{\mathcal{Q}}\left(\mathcal{E}_{k}\left(t_{j}, .\right)+\mathcal{D}_{k}\left(\tilde{q}_{j-1}, .\right)\right)+\frac{\varepsilon}{2}+\frac{\varepsilon}{6}
$$

for all $j=1, \ldots, N$ and $k$ sufficiently large.

Because $q_{j}^{k} \rightarrow \tilde{q}_{j}$ as $k \rightarrow \infty$ and the energies are bounded for $k$ sufficiently large (cf. (4.1)), the continuous convergence assumption (4.Г2) shows $\mathcal{D}_{k}\left(q_{j}^{k}, \tilde{q}_{j}\right) \rightarrow$ 0 and $\mathcal{D}_{k}\left(\tilde{q}_{j}, q_{j}^{k}\right) \rightarrow 0$ as $k \rightarrow \infty$, i.e.

$$
\max \left\{\mathcal{D}_{k}\left(q_{j}^{k}, \tilde{q}_{j}\right), \mathcal{D}_{k}\left(\tilde{q}_{j}, q_{j}^{k}\right)\right\} \leq \frac{\varepsilon}{6}
$$

for all $j=1, \ldots, N$ and $k$ sufficiently large.

So far we have constructed sequences and selected some $k_{0}=k_{0}(\varepsilon)$ large enough such that (4.1) and (4.2) are fulfilled for all $q_{j}^{k}$ with $k \geq k_{0}$. We still need to show that these $q_{j}^{k}$ form a discrete solution to $\left(\operatorname{AIP}_{k, \varepsilon}^{\Pi}\right)$.

For all $k \geq k_{0}$ and all $j=2, \ldots, N$, we find by the triangle inequality and estimate $(4.2)$

$$
\begin{aligned}
\inf _{\mathcal{Q}}\left(\mathcal{E}_{k}\left(t_{j}, .\right)+\mathcal{D}_{k}\left(\tilde{q}_{j-1}, .\right)\right) & \leq \inf _{\mathcal{Q}}\left(\mathcal{E}_{k}\left(t_{j}, .\right)+\mathcal{D}_{k}\left(q_{j-1}^{k}, .\right)\right)+\mathcal{D}_{k}\left(\tilde{q}_{j-1}, q_{j-1}^{k}\right) \\
& \leq \inf _{\mathcal{Q}}\left(\mathcal{E}_{k}\left(t_{j}, .\right)+\mathcal{D}_{k}\left(q_{j-1}^{k}, .\right)\right)+\frac{\varepsilon}{6} .
\end{aligned}
$$

In the case $j=1$, we have $q_{0}^{k}=\tilde{q}_{0}=q_{0}$ for all $k$ and hence (4.3) also holds for $j=1$.

Now, using first the triangle inequality, then (4.1) and (4.2), and finally (4.3), we deduce

$$
\begin{aligned}
\mathcal{E}_{k}\left(t_{j}, q_{j}^{k}\right)+\mathcal{D}_{k}\left(q_{j-1}^{k}, q_{j}^{k}\right) & \leq \mathcal{E}_{k}\left(t_{j}, q_{j}^{k}\right)+\mathcal{D}_{k}\left(\tilde{q}_{j-1}, q_{j}^{k}\right)+\mathcal{D}_{k}\left(q_{j-1}^{k}, \tilde{q}_{j-1}\right) \\
& \leq \inf _{\mathcal{Q}}\left(\mathcal{E}_{k}\left(t_{j}, .\right)+\mathcal{D}_{k}\left(\tilde{q}_{j-1}, .\right)\right)+\frac{\varepsilon}{2}+\frac{\varepsilon}{6}+\frac{\varepsilon}{6} \\
& \leq \inf _{\mathcal{Q}}\left(\mathcal{E}_{k}\left(t_{j}, .\right)+\mathcal{D}_{k}\left(q_{j-1}^{k}, .\right)\right)+\frac{\varepsilon}{2}+\frac{\varepsilon}{6}+\frac{\varepsilon}{6}+\frac{\varepsilon}{6} \\
& =\inf _{\mathcal{Q}}\left(\mathcal{E}_{k}\left(t_{j}, .\right)+\mathcal{D}_{k}\left(q_{j-1}^{k}, .\right)\right)+\varepsilon .
\end{aligned}
$$

But this is just $q_{j}^{k} \in \operatorname{Argmin}_{\varepsilon}\left(\mathcal{E}_{k}\left(t_{j},.\right)+\mathcal{D}_{k}\left(q_{j-1}^{k},.\right)\right)$ for $j=1, \ldots, N$, and the existence of solutions to $\left(\mathrm{AIP}_{k, \varepsilon}^{\Pi}\right)$ is shown for $k \geq k_{0}=k_{0}(\varepsilon)$. Trivially, we can fill up this sequence for $k<k_{0}$ with arbitrary solutions to $\left(\operatorname{AIP}_{k, \varepsilon}^{\Pi}\right)$. The claim $q_{j}^{k} \rightarrow \tilde{q}_{j}=q\left(t_{j}\right)$ is clear by the choice of the $q_{j}^{k}$ and Theorem 2.2.

To formulate the next result we have to strengthen the conditions on the sequential convergence on $\mathcal{Q}$, such that we are able to extract from a double sequence a suitable diagonal sequence. For $E \in \mathbb{R}$ we introduce the sublevel sets

$$
\Lambda(E)=\left\{q \in \mathcal{Q}: \text { there exists }(t, k) \in[0, T] \times \mathbb{N}_{\infty} \text { with } \mathcal{E}_{k}(t, q) \leq E\right\} .
$$


The assumption reads as follows.

$$
\begin{aligned}
& \text { There exists a metric } d: \mathcal{Q} \times \mathcal{Q} \rightarrow[0, \infty) \\
& \text { such that for all } E \in \mathbb{R} \text { and for all } q_{k} \in \Lambda(E), k \in \mathbb{N}_{\infty}: \\
& q_{k} \rightarrow q_{\infty} \text { if and only if } d\left(q_{k}, q_{\infty}\right) \rightarrow 0 .
\end{aligned}
$$

The final result states that every solution of the energetic system $\left(\mathcal{Q}, \mathcal{E}_{\infty}, \mathcal{D}_{\infty}\right)$ can be approximated by solutions of the approximate incremental problems $\left(\operatorname{AIP}_{k_{n}, \varepsilon_{n}}^{\Pi_{n}}\right)$, if the partitions $\Pi_{n}, \varepsilon_{n} \rightarrow 0$, and $k_{n} \rightarrow \infty$ are chosen suitably.

Theorem 4.3. Let the assumptions of Theorem 4.2 and the new assumption (4.5) hold. Moreover, let $q_{\infty}:[0, T] \rightarrow \mathcal{Q}$ be a solution to $\left(\mathrm{S}_{\infty}\right)$ \& $\left(\mathrm{E}_{\infty}\right)$ with initial value $q_{0}=q_{\infty}(0) \in \mathcal{S}_{\infty}(0)$. Then, for every sequence $\varepsilon_{n} \rightarrow 0$, there exists a sequence of partitions $\Pi_{n}=\left(0, t_{1}^{n}, \ldots, t_{N(n)-1}^{n}, T\right)$ of $[0, T]$ with $\left\|\Pi_{n}\right\| \rightarrow 0$ as $n \rightarrow \infty$, a sequence $\left(k_{n}\right)_{n}$ of problem indices with $k_{n} \rightarrow \infty$ as $n \rightarrow \infty$, and discrete solution $q_{k_{n}}^{\Pi_{n}}:=\left(q_{0}^{k_{n}}, q_{1}^{k_{n}}, \ldots, q_{N(n)}^{k_{n}}\right)$, defined on the partition $\Pi_{n}$, of the approximate incremental problem $\left(\mathrm{AIP}_{k_{n}, \varepsilon_{n}}^{\Pi_{n}}\right)$ associated with $\mathcal{E}_{k_{n}}$ and $\mathcal{D}_{k_{n}}$ such that the piecewise constant interpolants $\bar{q}_{n}:[0, T] \rightarrow \mathcal{Q}$ of these discrete solutions converge on a dense subset $\mathcal{T}$ of $[0, T]$ to the solution $q_{\infty}$.

Proof. We use a sequence of nested partitions $\Pi_{n} \subseteq \Pi_{n+1}$ with $\left\|\Pi_{n}\right\| \leq \varepsilon_{n} /\left(2 c_{\mathrm{R}}\right)$, where $c_{\mathrm{R}}=c_{\mathrm{R}}\left(q_{0}\right)>0$ is the reverse approximation constant from Theorem 2.2. Then, $\mathcal{T}:=\bigcup_{n \in \mathbb{N}} \Pi_{n}$ is dense in $[0, T]$.

Applying Theorem 2.2 we find, for all $n \in \mathbb{N}$, a sequence $\left(\left(q_{j}^{n, k}\right)_{j=0, \ldots, N(n)}\right)_{k \in \mathbb{N}}$ in $\mathcal{Q}^{N(n)}$ of solutions to $\left(\operatorname{AIP}_{k, \varepsilon_{n}}^{\Pi_{n}}\right)$, such that

$$
q_{j}^{n, k} \rightarrow q\left(t_{j}^{n}\right), \mathcal{E}\left(t_{j}^{n}, q_{j}^{n, k}\right) \rightarrow \mathcal{E}\left(t_{j}^{n}, q\left(t_{j}^{n}\right)\right) \quad \text { for all } \quad n \in \mathbb{N} j=0, \ldots, N(n) .
$$

With $E:=\sup \left\{\mathcal{E}_{\infty}(t, q(t)): t \in[0, T]\right\}<\infty$ (cf. [19]), we find $K(n) \in \mathbb{N}$, such that $\max \left\{\mathcal{E}_{k}\left(t_{j}^{n}, q_{j}^{n, k}\right): j=0,1, \ldots, N(n)\right\} \leq E+1$ for all $k \geq K(n)$. Thus, we can employ assumption (4.5) on the set $\Lambda(E+1)$ and obtain, for fixed $n \in \mathbb{N}$,

$$
\delta(n, k)=\max \left\{d\left(q_{j}^{n, k}, q\left(t_{j}^{n}\right)\right): j=0,1, \ldots, N(n)\right\} \rightarrow 0 \quad \text { for } \quad k \rightarrow \infty .
$$

Choose the subsequence $\left(k_{n}\right)_{n \in \mathbb{N}}$ such that $k_{n} \geq K(n)$ and $\delta\left(n, k_{n}\right) \leq 1 / n$ and define the solutions $\left(q_{0}^{k_{n}}, \ldots, q_{N(n)}^{k_{n}}\right)$ via $q_{j}^{k_{n}}:=q_{j}^{n, k_{n}}$. Since the sequence of partitions is nested, for each $t \in \mathcal{T}$ there exists an $m(t)$ such that $t \in \Pi_{n}$ for $n \geq m(t)$, i.e., $t=t_{J(t, n)}^{n}$. Thus,

$$
d\left(\bar{q}_{n}(t), q(t)\right)=d\left(q_{J(t, n)}^{n, k_{n}}, q\left(t_{J(t, n)}^{n}\right)\right) \leq \delta\left(n, k_{n}\right) \leq 1 / n \quad \text { for } \quad n \geq m(t) .
$$

This is the desired convergence result, and the theorem is established.

Remark 4.4. The convergence result in Theorem 4.3 can be strengthened to convergence for all $t \in[0, T]$. One first uses the ideas in the proof of Helly's selection principle (cf. e.g. $[16,24])$ to show that $\bar{z}_{n}(t) \rightarrow z_{\infty}(t)$ for all $t$. For this one employs the uniform a priori bound on the dissipation and includes all jump points 
of $z_{\infty}$ into $\mathcal{T}$. Next, one needs to impose the further assumption that the global minimizer $\phi=\Phi(t, z)$ of $\mathcal{E}(t, \cdot, z)$ is unique. Then, it can be shown $\bar{\phi}_{n}(t) \rightarrow \phi(t)$ as well, cf. [16].

Just like in Section 2, we cannot expect strict approximability of solutions to $\left(\mathrm{S}_{\infty}\right) \&\left(\mathrm{E}_{\infty}\right)$ by discrete solutions of $\left(\mathrm{IP}_{k}^{\Pi}\right)$ instead of $\left(\mathrm{AIP}_{k, \varepsilon}^{\Pi}\right)$. This, in fact, has been settled already in Counterexample 2.4, because the latter shows that even for a constant sequence of functionals, we cannot get strict approximability.

To conclude this section, we further show that one cannot expect approximability of solutions to $\left(\mathrm{S}_{\infty}\right) \&\left(\mathrm{E}_{\infty}\right)$ by time-continuous solutions to $\left(\mathrm{S}_{k}\right) \&\left(\mathrm{E}_{k}\right)$ instead of approximate time-incremental solutions to $\left(\mathrm{AIP}_{k, \varepsilon}^{\Pi}\right)$.

Counterexample 4.5. Consider the state space $\mathcal{Q}=[0,1]$, the time interval $[0, T]=$ $[0,2]$ and the energy functionals

$$
\mathcal{E}_{\infty}(t, q):=-q \text { and } \mathcal{E}_{k}(t, q):=\frac{q^{2}}{2 k}-q \quad \text { for } \quad k \in \mathbb{N}
$$

for $t \in[0,2]$ and $q \in \mathbb{R}$. Also, choose $\mathcal{D}_{k}\left(q_{1}, q_{2}\right)=\mathcal{D}_{\infty}\left(q_{1}, q_{2}\right):=\mathcal{D}\left(q_{1}, q_{2}\right):=$ $\left|q_{2}-q_{1}\right|$. As initial value we select $q_{0}=0$. This setting can be seen as a degenerately convex problem in the limit $k=\infty$ with strictly convex approximations for $k \in \mathbb{N}$. The process

$$
q_{\infty}(t):= \begin{cases}0 & \text { if } \quad t \in[0,1) \\ 1 & \text { if } t \in[1,2]\end{cases}
$$

is one of the many solutions of the rate-independent formulation associated with $\mathcal{E}_{\infty}$ and $\mathcal{D}$. The stable states $\mathcal{S}_{\infty}(t)$ are easily seen to be the whole space, i.e. $\mathcal{S}_{\infty}(t)=\mathcal{S}_{\infty}(0)=[0,1]$, thus the stability condition is trivially fulfilled. For $t \in$ $[0,1)$, the energy balance is trivial and for $t \in[1,2]$ we have

$$
\begin{aligned}
\mathcal{E}_{\infty}\left(t, q_{\infty}(t)\right)+\operatorname{Diss}_{\mathcal{D}}\left(q_{\infty} ;[0, t]\right) & =\mathcal{E}_{\infty}(t, 1)+\mathcal{D}(0,1)=-1+1=0 \\
& =\mathcal{E}_{\infty}\left(0, q_{0}\right)+\int_{0}^{t} \partial_{t} \mathcal{E}_{\infty}\left(\tau, q_{\infty}(\tau)\right) \mathrm{d} \tau
\end{aligned}
$$

Hence, $q_{\infty}$ is an energetic solution of $\left(\mathrm{S}_{\infty}\right) \&\left(\mathrm{E}_{\infty}\right)$. We now show that $q_{\infty}$ cannot be approximated by solutions to $\left(\mathrm{S}_{k}\right) \&\left(\mathrm{E}_{k}\right)$.

For all $k \in \mathbb{N}$, the stable sets $\mathcal{S}_{k}(t)=\mathcal{S}_{k}(0)$ again are the whole space $[0,1]$, since it holds for all $q, \hat{q} \in[0,1]$ that

$$
\begin{aligned}
& \mathcal{E}_{k}(t, \hat{q})+\mathcal{D}(q, \hat{q})-\mathcal{E}_{k}(t, q) \\
& \quad=\frac{\hat{q}^{2}-q^{2}}{2 k}+(q-\hat{q})+|\hat{q}-q| \\
& \quad=\left\{\begin{array}{ll}
\left(\hat{q}^{2}-q^{2}\right) /(2 k) & \text { if } \hat{q} \geq q \\
(q-\hat{q})(2-(\hat{q}+q) /(2 k)) \geq(q-\hat{q})\left(2-k^{-1}\right) & \text { if } \hat{q}<q
\end{array}\right\} \geq 0,
\end{aligned}
$$

i.e. $q \in \mathcal{S}_{k}(t)$. Now, the zero-process $q_{k} \equiv 0$ trivially is a solution of $\left(\mathrm{S}_{k}\right) \&\left(\mathrm{E}_{k}\right)$ and because the problem is strictly convex and the stable sets are convex, we immediately get the uniqueness of this solution [19, Theorem 4.2]. But the zeroprocess does not approximate $q_{\infty}$ in any reasonable sense. 


\section{Quantitative backward error analysis}

In this section we use the shorthand $\mathcal{E}=\mathcal{E}_{\infty}$ and $\mathcal{D}=\mathcal{D}_{\infty}$. Moreover, without loss of generality, we assume that the energies $\mathcal{E}(t, q)$ and $\mathcal{E}_{k}(t, q)$ are uniformly bounded from below by a positive constant. Hence, we may choose the constant $c_{0}^{E}$ to be 0 .

The aim of this section is twofold. Under additional quantitative continuity assumptions we prove exact estimates for the reverse approximation. Using this we then provide an example where the $\Gamma$-convergence is realized as numerical approximation via Galerkin subspaces $V_{k} \subseteq \mathcal{Q}$, where $\mathcal{Q}$ now is a Banach space and the projections $P_{k}: \mathcal{Q} \rightarrow V_{k} \subseteq \mathcal{Q}$ satisfy $P_{k} q \rightarrow q$ for all $q \in \mathcal{Q}$.

We consider the limit functionals $\mathcal{E}:[0, T] \times \mathcal{Q} \rightarrow \mathbb{R}$ and $\mathcal{D}: \mathcal{Q} \times \mathcal{Q} \rightarrow[0, \infty)$ as above. Moreover, we have functionals $\mathcal{E}_{k}$ and $\mathcal{D}_{k}$ such that

$$
\mathcal{E}_{k}(t, q)=+\infty \quad \text { for } \quad q \in \mathcal{Q} \backslash V_{k} .
$$

On the other hand, the main assumptions involve continuity properties of $\mathcal{E}$ and $\mathcal{D}$ :

$$
\begin{aligned}
|\mathcal{E}(t, q)-\mathcal{E}(t, \hat{q})| & \leq \frac{c}{2}(\mathcal{E}(t, q)+\mathcal{E}(t, \hat{q}))\|q-\hat{q}\|, \\
\left|\mathcal{D}\left(q_{0}, q_{1}\right)-\mathcal{D}\left(\hat{q}_{0}, \hat{q}_{1}\right)\right| & \leq c\left(\left\|q_{0}-\hat{q}_{0}\right\|+\left\|q_{1}-\hat{q}_{1}\right\|\right)
\end{aligned}
$$

Further, we assume quantified estimates on the approximations. For all $q, \tilde{q} \in$ $V_{k}$ and all $t \in[0, T]$ we have an $\alpha_{k}>0$ such that

$$
\begin{aligned}
\left|\mathcal{E}_{k}(t, q)-\mathcal{E}(t, q)\right| & \leq \mathcal{E}(t, q) \alpha_{k} \\
\left|\mathcal{D}_{k}\left(q_{0}, q_{1}\right)-\mathcal{D}\left(q_{0}, q_{1}\right)\right| & \leq \frac{\alpha_{k}}{2}\left(\mathcal{E}\left(t, q_{0}\right)+\mathcal{E}\left(t, q_{1}\right)\right)
\end{aligned}
$$

Lemma 5.1. Let $q:[0, T] \rightarrow \mathcal{Q}$ be given such that

$$
\begin{aligned}
& E_{q}=\sup \{\mathcal{E}(t, q(s)): s, t \in[0, T]\}<\infty, \\
& \delta_{k}=\sup \left\{\left\|P_{k} q(t)-q(t)\right\|: s, t \in[0, T]\right\}<\infty
\end{aligned}
$$

Moreover, assume $\alpha_{k} \leq 1$ and $c \delta_{k} \leq 1 / 2$. Then,

$$
\begin{aligned}
& \sup \left\{\mathcal{E}\left(t, P_{k} q(s)\right): s, t \in[0, T]\right\} \leq 2 E_{q}, \\
& \sup \left\{\mathcal{E}_{k}\left(t, P_{k} q(s)\right): s, t \in[0, T]\right\} \leq 4 E_{q} .
\end{aligned}
$$

Proof. Using (5.1) we have for $\hat{q}:=P_{k} q(s)$

$$
\mathcal{E}(t, \hat{q})-\mathcal{E}(t, q(s)) \leq \frac{c}{2}(\mathcal{E}(t, q(s))+\mathcal{E}(t, \hat{q})) \delta_{k},
$$

which implies

$$
\mathcal{E}(t, \hat{q}) \leq \frac{1+c \delta_{k} / 2}{1-c \delta_{k} / 2} \mathcal{E}(t, q(s)) \leq \frac{5}{3} E_{q}
$$

Hence, (5.5) is established and (5.6) follows by applying (5.3) to $q=P_{k} q(s)$. 
To simplify notation in the proof of the main result of this section (cf. Theorem 5.3), we introduce

$$
\iota_{k}(t, q):=\inf \left\{\mathcal{E}_{k}(t, \hat{q})+\mathcal{D}_{k}(q, \hat{q}): \hat{q} \in \mathcal{Q}\right\}
$$

and similarly for the limit functionals $\mathcal{E}$ and $\mathcal{D}$. The next result is a quantitative version of Lemma 2.1 .

Lemma 5.2. Let $q:[0, T] \rightarrow \mathcal{Q}$ be a solution to the system associated with the functionals $\mathcal{E}$ and $\mathcal{D}$, where $\mathcal{E}$ satisfies (4.E1). Then, for all $s, t \in[0, T]$ we have

$$
\iota(s, q(s)) \leq \mathrm{e}^{c_{1}^{E}|t-s|} \iota(t, q(s)) .
$$

Moreover, if $\Pi=\left(0=t_{0}, t_{1}, \ldots, t_{N}=T\right)$ is a partition, then $\left(q\left(t_{j}\right)\right)_{j}$ solves $\left(\operatorname{AIP}_{\varepsilon(\Pi)}^{\Pi}\right)$ with

$$
\varepsilon(\Pi)=2\left(\mathrm{e}^{c_{1}^{E}\|\Pi\|}-1\right) E_{q} .
$$

Proof. For estimate (5.7) choose $q_{\rho}$ in $\operatorname{Argmin}_{\rho} \mathcal{E}(t,)+.\mathcal{D}(q(s),$.$) . Then, we have$

$$
\begin{aligned}
\iota(s, q(s)) & =\mathcal{E}(s, q(s)) \leq \mathcal{E}\left(s, q_{\rho}\right)+\mathcal{D}\left(q(s), q_{\rho}\right) \\
& \leq \mathrm{e}^{c_{1}^{E}|t-s|}\left(\mathcal{E}\left(t, q_{\rho}\right)+\mathcal{D}\left(q(s), q_{\rho}\right)\right) \leq \mathrm{e}^{c_{1}^{E}|t-s|}(\iota(t, q(s))+\rho),
\end{aligned}
$$

where the first estimate uses stability of $q(s)$, the second follows from (2.1), and the third is the definition of $q_{\rho}$. For $\rho \rightarrow 0$ we obtain estimate (5.7).

Using the energy balance (E) and $(2.2)$ we find with $\iota_{j}:=\iota\left(t_{j}, q_{j}\right)=\mathcal{E}\left(t_{j}, q\left(t_{j}\right)\right)$,

$$
\begin{aligned}
\mathcal{E}\left(t_{j}, q_{j}\right)+\mathcal{D}\left(q_{j-1}, q_{j}\right) & \leq \mathcal{E}\left(t_{j}, q_{j}\right)+\operatorname{Diss}_{\mathcal{D}}\left(q ;\left[t_{j-1}, t_{j}\right]\right) \\
& =\mathcal{E}\left(t_{j-1}, q_{j-1}\right)+\int_{t_{j-1}}^{t_{j}} \partial_{t} \mathcal{E}(\tau, q(\tau)) \mathrm{d} \tau \\
& \leq \iota_{j-1}+\int_{t_{j-1}}^{t_{j}} c_{1}^{E} \mathrm{e}^{c_{1}^{E}\left(\tau-t_{j-1}\right)} E_{q} \mathrm{~d} \tau .
\end{aligned}
$$

Using (5.7) with $s=t_{j-1}$ and $t=t_{j}$ we proceed to get

$$
\mathcal{E}\left(t_{j}, q_{j}\right)+\mathcal{D}\left(q_{j-1}, q_{j}\right) \leq \mathrm{e}^{c_{1}^{E}\left(t_{j}-t_{j-1}\right)} \iota\left(t_{j}, q_{j-1}\right)+\left(\mathrm{e}^{c_{1}^{E}\left(t_{j}-t_{j-1}\right)}-1\right) E_{q} .
$$

Using $\iota\left(t_{j}, q_{j-1}\right) \leq \mathcal{E}\left(t_{j}, q\left(t_{j-1}\right)\right) \leq E_{q}$ and $t_{j}-t_{j-1} \leq\|\Pi\|$, we obtain

$$
\mathcal{E}\left(t_{j}, q_{j}\right)+\mathcal{D}\left(q_{j-1}, q_{j}\right) \leq \iota\left(t_{j}, q_{j-1}\right)+2\left(\mathrm{e}^{c_{1}^{E}\|\Pi\|}-1\right) E_{q},
$$

and (5.8) is established.

Theorem 5.3. Let $q:[0, T] \rightarrow \mathcal{Q}$ be a solution to the energetic system $(\mathcal{Q}, \mathcal{E}, \mathcal{D})$. Let the assumptions of Section 4 as well as the estimates (5.1)-(5.4) hold and let $\delta_{k}$ and $E_{q}$ be defined as in Lemma 5.1. Then, for all $k \in \mathbb{N}$ such that $c \delta_{k} \leq 1 / 2$ and $\alpha_{k} \leq 1$, and all partitions $\Pi=\left(0=t_{0}, t_{1}, \ldots, t_{N}=T\right)$ of $[0, T]$, the sequences $\left(P_{k} q\left(t_{j}\right)\right)_{j=0, \ldots, N}$ are solutions to $\left(\mathrm{AIP}_{k, \hat{\varepsilon}(k, \Pi)}^{\Pi}\right)$ with

$$
\hat{\varepsilon}(k, \Pi)=E_{q}\left[2\left(\mathrm{e}^{c_{1}^{E}\|\Pi\|}-1\right)+11 \alpha_{k}+5 c \delta_{k}\right] .
$$


Proof. For short notation let $q_{j}^{k}=P_{k} q\left(t_{j}\right)$ and $q_{j}=q\left(t_{j}\right)$. We use the assumptions (5.3) and (5.4) and the a-priori estimates (5.5) and (5.6) to estimate as follows:

$$
\begin{aligned}
\mathcal{E}_{k}\left(t_{j}, q_{j}^{k}\right)-\mathcal{D}_{k}\left(q_{j-1}^{k}, q_{j}^{k}\right) & \leq \mathcal{E}\left(t_{j}, q_{j}^{k}\right)+\mathcal{D}\left(q_{j-1}^{k}, q_{j}^{k}\right)+4 E_{q} \alpha_{k} \\
& \leq \mathcal{E}\left(t_{j}, q_{j}\right)+\mathcal{D}\left(q_{j-1}, q_{j}\right)+E_{q}\left(4 \alpha_{k}+3 c \delta_{k}\right) .
\end{aligned}
$$

Next we estimate $\iota_{k}\left(t_{j}, q_{j-1}^{k}\right)$ from below using $\iota\left(t_{j}, q_{j-1}\right)$. For this let $\rho_{0}:=$ $\mathcal{E}_{k}\left(t_{j}, q_{j-1}^{k}\right)-\iota_{k}\left(t_{j}, q_{j-1}^{k}\right)$, which implies $\rho_{0} \geq 0$. If $\rho_{0}=0$, then

$$
\begin{aligned}
\iota_{k}\left(t_{j}, q_{j-1}^{k}\right)= & \mathcal{E}_{k}\left(t_{j}, q_{j-1}^{k}\right) \geq \mathcal{E}\left(t_{j}, q_{j-1}^{k}\right)-2 E_{q} \alpha_{k} \\
\geq & \mathcal{E}\left(t_{j}, q_{j-1}\right)-E_{q}\left(2 \alpha_{k}+2 c \delta_{k}\right) \geq \iota\left(t_{j}, q_{j-1}\right) \\
& -E_{q}\left(2 \alpha_{k}+2 c \delta_{k}\right) .
\end{aligned}
$$

If $\rho_{0}>0$, we find, for each $\rho \in\left(0, \rho_{0}\right)$, a $q_{\rho} \in V_{k}$ with $\mathcal{E}_{k}\left(t_{j}, q_{\rho}\right)+\mathcal{D}_{k}\left(q_{j-1}^{k}, q_{\rho}\right) \leq$ $\mathcal{E}_{k}\left(t_{j}, q_{j-1}^{k}\right) \leq 4 E_{q}$ and $q_{\rho} \in \operatorname{Argmin}_{\rho}\left(\mathcal{E}_{k}\left(t_{j},.\right)+\mathcal{D}_{k}\left(q_{j-1}^{k},.\right)\right.$. Hence, we estimate

$$
\begin{aligned}
\iota_{k}\left(t_{j}, q_{j-1}^{k}\right) & \geq-\rho+\mathcal{E}_{k}\left(t_{j}, q_{\rho}\right)+\mathcal{D}_{k}\left(q_{j-1}^{k}, q_{\rho}\right) \\
& \geq-\rho+\mathcal{E}\left(t_{j}, q_{\rho}\right)+\mathcal{D}\left(q_{j-1}^{k}, q_{\rho}\right)-7 E_{q} \alpha_{k} \\
& \geq-\rho+\mathcal{E}\left(t_{j}, q_{\rho}\right)+\mathcal{D}\left(q_{j-1}, q_{\rho}\right)-E_{q}\left(7 \alpha_{k}+c \delta_{k}\right) \\
& \geq-\rho+\iota\left(t_{j}, q_{j-1}\right)-E_{q}\left(7 \alpha_{k}+c \delta_{k}\right) .
\end{aligned}
$$

Taking the limit $\rho \searrow 0$ and combining with the case $\rho_{0}=0$, see (5.10), we find

$$
\iota\left(t_{j}, q_{j-1}\right) \leq \iota_{k}\left(t_{j}, q_{j-1}^{k}\right)+E_{q}\left(7 \alpha_{k}+2 c \delta_{k}\right) .
$$

We conclude by noting that Lemma 5.2 gives

$$
\mathcal{E}\left(t_{j}, q_{j-1}\right)+\mathcal{D}\left(q_{j-1}, q_{j}\right) \leq \iota\left(t_{j}, q_{j-1}\right)+\varepsilon(\Pi)
$$

with $\varepsilon(\Pi)$ defined in (5.8). Combining this with (5.9) and (5.11) we have

$$
\mathcal{E}_{k}\left(t_{j}, q_{j-1}^{k}\right)+\mathcal{D}_{k}\left(q_{j-1}^{k}, q_{j}^{k}\right) \leq \iota_{k}\left(t_{j}, q_{j-1}^{k}\right)+\varepsilon(\Pi)+E_{q}\left(11 \alpha_{k}+5 c \delta_{k}\right),
$$

which gives the desired result for $\hat{\varepsilon}(k, \Pi)$.

We consider an example for phase transitions on a smooth, bounded domain $\Omega \subseteq \mathbb{R}^{d}$ with $d \geq 2$. The state space is $\mathcal{Q}=\mathcal{Z}=\mathrm{H}^{1}(\Omega)$ with dissipation distance

$$
\mathcal{D}\left(q_{0}, q_{1}\right):=\left\|q_{1}-q_{0}\right\|_{\mathrm{L}^{1}(\Omega)} .
$$

The energy functional takes the form

$$
\mathcal{E}(t, q):=\int_{\Omega} \frac{1}{2}|\nabla q(x)|^{2}+G(q(x))-f(t, x) q(x) \mathrm{d} x,
$$

where the loading $f$ satisfies $f \in \mathrm{C}^{1}([0, T] \times \bar{\Omega})$. The potential $G \in \mathrm{C}^{2}(\mathbb{R} ; \mathbb{R})$ has a bounded second derivative $G^{\prime \prime}$ and is coercive, i.e., there is $C>0$ such that $G(q) \geq q^{2} / C-C$. It is important to note that $G$ may be nonconvex, such that also $\mathcal{E}(t,):. \mathcal{Q} \rightarrow \mathbb{R}$ may be nonconvex. Thus, in general, the energetic solutions for the functionals $\mathcal{E}$ and $\mathcal{D}$ will not be continuous in time (even for the $\mathrm{L}^{1}$-norm). 
Moreover, uniqueness of solutions under an initial condition $q(0)=q_{0}$ cannot be expected, cf. [19].

The classical existence results apply (see $[16,23])$ giving solutions

$$
q \in \mathrm{L}^{\infty}\left([0, T] ; \mathrm{H}^{1}(\Omega)\right) \cap \mathrm{BV}\left([0, T] ; \mathrm{L}^{1}(\Omega)\right)
$$

for each stable initial datum $q_{0} \in \mathrm{H}^{1}(\Omega)$. However, the stability condition $q(t) \in$ $\mathcal{S}(t)$ gives the variational inequality

$$
\Delta q-G^{\prime}(q)+f(t, \cdot) \in \partial \operatorname{Sign}(0)=[-1,1] .
$$

We assume that the domain is a convex polytope and that $f$ is bounded. Then, elliptic regularity implies

$$
q \in \mathrm{L}^{\infty}\left([0, T] ; \mathrm{H}^{2}(\Omega)\right) .
$$

Our application of $\Gamma$-convergence relates to a sequence of numerical approximations, as is discussed in much greater detail in [21]. For this we choose a sequences $\left(\mathcal{T}_{k}\right)_{k}$ of triangulations, such that the maximal diameters

$$
h_{k}=\rho\left(\mathcal{T}_{k}\right) \quad \text { with } \quad \rho(\mathcal{T}):=\max \{\operatorname{diam}(T): T \in \mathcal{T}\}
$$

tend to 0 . However, as our estimates are quantitative, we give estimates for all triangulations. Thus, we simplify the notation by using the subscript $\mathcal{T}$ instead of $k$.

Let $V_{\mathcal{T}} \subset \mathcal{Q}=\mathrm{H}^{1}(\Omega)$ be a space of continuous, piecewise linear functions (finite-element space) associated with $\mathcal{T}$. By $P_{\mathcal{T}}$ we denote the $\mathrm{H}^{1}$-orthogonal projection of $\mathcal{Q}$ onto $V_{\mathcal{T}}$, which satisfies

$$
P_{\mathcal{T}} q \rightarrow q \text { in } \mathrm{H}^{1}(\Omega) \text { for } \rho(\mathcal{T}) \rightarrow 0,
$$

There exists $C>0$ such that for all $q \in \mathrm{H}^{2}(\Omega):\left\|P_{\mathcal{T}} q-q\right\|_{\mathcal{Q}} \leq C \rho(\mathcal{T})\|q\|_{\mathrm{H}^{2}(\Omega)}$.

For any given $\mathcal{T}$ we define

$$
\begin{aligned}
\mathcal{D}_{\mathcal{T}}(q, \hat{q}) & :=\mathcal{D}(q, \hat{q}), \\
\mathcal{E}_{\mathcal{T}}(t, q) & :=\int_{\Omega} \frac{1}{2}|\nabla q|^{2} \mathrm{~d} x+\sum_{T \in \mathcal{T}} \frac{\operatorname{vol} T}{d+1} \sum_{j=0}^{d}\left[G\left(q\left(X_{j}^{T}\right)\right)-f\left(t, X_{j}^{T}\right) q\left(X_{j}^{T}\right)\right],
\end{aligned}
$$

where $\left(X_{j}^{T}\right)_{j=0, \ldots, d}$ are the vertices of the tetrahedron $T \in \mathcal{T}$. For $q \in V_{\mathcal{T}}$ one has

$$
\left|\mathcal{E}_{\mathcal{T}}(t, q)-\mathcal{E}(t, q)\right| \leq \mathcal{E}(t, q) \alpha(\mathcal{T}),
$$

where the class of triangulations must be restricted in such a way that $\alpha(\mathcal{T}) \rightarrow 0$ for $\rho(\mathcal{T}) \rightarrow 0$. For instance, for quasi-uniform meshes one has $\alpha(\mathcal{T}) \leq C_{\text {qu }} \rho(\mathcal{T})^{2}$, where the constant $C_{\mathrm{qu}}$ only depends on the lower bound of the interior angles.

Thus, (5.3) and (5.4) hold. Of course, (5.1) and (5.2) hold trivially. Moreover, $\delta_{k}$ in Lemma 5.1 is given as

$$
\delta_{\mathcal{T}}=\sup \left\{\left\|P_{\mathcal{T}} q(t)-q(t)\right\|: t \in[0, T]\right\} \leq C \rho(\mathcal{T})\|q\|_{\mathrm{L}^{\infty}\left([0, T] ; \mathrm{H}^{2}(\Omega)\right)} .
$$

As a result, we obtain the following reverse approximation result which can be seen as a type of justification of a backward error analysis of space-time discretization. 
Theorem 5.4. Let $\mathcal{Q}=\mathrm{H}^{1}(\Omega)$ and $\mathcal{E}, \mathcal{D}$ be as given in (5.12) and (5.13) with $G$ and $f$ as specified. Let $q:[0, T] \rightarrow \mathcal{Q}$ solve the rate-independent energetic system $(\mathcal{Q}, \mathcal{E}, \mathcal{D})$. Then, there exists a constant $C_{*}$ such that the following holds: If $\Pi=\left(0=t_{0}, t_{1}, \ldots, t_{N}=T\right)$ is a partition of $[0, T]$ with fineness $\|\Pi\|$ and $\mathcal{T}$ is a triangulation of $\Omega$ with $\|\Pi\|+\rho(\mathcal{T})+\alpha(\mathcal{T}) \leq 1 / C_{*}$, then the sequence $\left(q_{j}^{\mathcal{T}}\right)_{j=0, \ldots, N}$ defined via $q_{j}^{\mathcal{T}}:=P_{\mathcal{T}} q\left(t_{j}\right)$ is a solution to $\left(\operatorname{AIP}_{\mathcal{T}, \varepsilon}^{\Pi}\right)$ with $\varepsilon=C_{*}(\|\Pi\|+\rho(\mathcal{T})+$ $\alpha(\mathcal{T}))$ and satisfies $\left\|q_{j}^{\mathcal{T}}-q\left(t_{j}\right)\right\|_{\mathcal{Q}} \leq C_{*} \rho(\mathcal{T})$ for $j=0,1, \ldots, N$.

\section{Regularization and relaxation}

\subsection{An example with a regularized functional}

Let the state space $\mathcal{Q}$ be the Sobolev space $\mathrm{H}^{1}(0,1)$ equipped with its weak topology. Consider the functionals

$$
\begin{aligned}
& \mathcal{E}_{k}(t, z):=\int_{0}^{1} \frac{1}{k}\left(z^{\prime \prime}(x)\right)^{2}+W\left(z^{\prime}(x)\right)+G(z(x))-f(t, x) z(x) \mathrm{d} x, \\
& \mathcal{E}_{\infty}(t, z):=\int_{0}^{1} W^{* *}\left(z^{\prime}(x)\right)+G(z(x))-f(t, x) z(x) \mathrm{d} x,
\end{aligned}
$$

where $f:[0, T] \times[0,1] \rightarrow \mathbb{R}$ is a prescribed loading $G$ is as in the previous section. The double-well potential is given via $W(s):=\min \left\{(s-1)^{2},(s+1)^{2}\right\}$ and has the convexification $W^{* *}$ with $W^{* *}(s)=W(s)$ for $|s| \geq 1$ and $W^{* *}(s)=0$ on $[-1,1]$. In order to apply $\mathcal{E}_{k}$, we need twice (weak) differentiability of $z$, which is only given in the subspace $\mathrm{H}^{2}(0,1)$. We therefore set $\mathcal{E}_{k}:=+\infty$ on $\mathrm{H}^{1}(0,1) \backslash \mathrm{H}^{2}(0,1)$. Hence, $\mathcal{E}_{k}$ can be seen as a regularization of $\mathcal{E}$ given via

$$
\mathcal{E}(t, z):=\int_{0}^{1} W\left(z^{\prime}(x)\right)+G(z(x))-f(t, x) z(x) \mathrm{d} x,
$$

which is not weakly lower semi-continuous on $\mathrm{H}^{1}(0,1)$. All the $\mathcal{E}_{k}$ have closed and bounded sublevels in $\mathrm{H}^{2}(0,1)$. Owing to the compact embedding $\mathrm{H}^{2}(0,1) \stackrel{c}{\hookrightarrow}$ $\mathrm{H}^{1}(0,1)$, these sublevels are compact in the weak topology of $\mathrm{H}^{1}(0,1)$.

Further, for all $k$ we use the $\mathrm{L}^{1}(0,1)$-norm as dissipation distance, i.e. $\mathcal{D}(u, v):=\|v-u\|_{1}$. Thus, solutions to the rate-independent energetic system $\left(\mathcal{Q}, \mathcal{E}_{k}, \mathcal{D}\right)$ exist by the standard results of the theory $[12,16,19]$ and satisfy the differential inclusion

$0 \in \operatorname{Sign}\left(\partial_{t} z\right)+\frac{1}{k} \partial_{x}^{4} z-\partial_{x}\left(\mathrm{D} W\left(\partial_{x} z\right)\right)+G^{\prime}(z)-f(t,$.$) \quad a.e. in \quad(t, x) \in[0, T] \times \Omega$

together with a smooth stable initial condition $z(0,)=.z_{0} \in \mathrm{H}^{2}(0,1)$. Similarly, solutions to the problem associated with $\mathcal{E}_{\infty}$ and $\mathcal{D}$ satisfy

$$
0 \in \operatorname{Sign}\left(\partial_{t} z\right)-\partial_{x}\left(D W^{* *}\left(\partial_{x} z\right)\right)+G^{\prime}(z)-f(t, .) \quad \text { a.e. in } \quad(t, x) \in[0, T] \times \Omega .
$$


It is well-known that the $\mathcal{E}_{k} \Gamma$-converge to $\mathcal{E}_{\infty}$ and all assumptions on the $\mathcal{E}_{k}$ are also easily seen to hold for adequately chosen loadings $f[7,26]$. Further, as noted in Example 4.1, the constant sequence of dissipation distances converges in the required sense.

From the results in [24] we know that solutions to the incremental problem $\left(\mathrm{IP}_{k}^{\Pi}\right)$ for the $k$ th problem admit a subsequence converging to a solution of $\left(\mathrm{S}_{\infty}\right) \&\left(\mathrm{E}_{\infty}\right)$. Now, the results of Section 4 imply that every solution to $\left(\mathrm{S}_{\infty}\right)$ $\&\left(\mathrm{E}_{\infty}\right)$ can be approximated by solutions to $\left(\mathrm{AIP}_{k, \varepsilon}^{\Pi}\right)$.

\subsection{An example for relaxation}

We might encounter energy functionals $\mathcal{E}: \mathcal{Q} \rightarrow \mathbb{R}_{\infty}$ for which an infimizing sequence converges, but the limit is no minimizer of $\mathcal{E}$. Such functionals $\mathcal{E}$ cannot have closed sublevels, i.e. they are not lower semicontinuous. In applications, this situation is caused by the development of microstructure [26,27]. In order to analyze the macroscopic behavior of minimizers of such functionals, we can "relax" $\mathcal{E}$ to its lower semicontinuous envelope $\mathcal{E}^{* *}: \mathcal{Q} \rightarrow \mathbb{R}_{\infty}$ and study the problem associated with the new functional $\mathcal{E}^{* *}$. The framework of $\Gamma$-convergence is designed in such a way that if we take the $\Gamma$-limit of the constant sequence $(\mathcal{E})_{k}$, we arrive at the relaxation $\mathcal{E}^{* *}$ of $\mathcal{E}[4,8]$. Thus, we can apply the methods developed above in order to understand the connection between the original and the relaxed problem.

In [24] it is shown that the relaxed problem is not "too small", i.e. a sequence of solutions to the approximate incremental problem $\left(\mathrm{AIP}_{\varepsilon}^{\Pi}\right)$ for the original energy functional $\mathcal{E}$ admits a limit point, which is an energetic solution to $\left(\mathrm{S}^{* *}\right) \&\left(\mathrm{E}^{* *}\right)$ for the relaxed functional $\mathcal{E}^{* *}$. In this work, we have shown that the relaxed problem also is not "too big", i.e. for every solution of $\left(\mathrm{S}^{* *}\right) \&\left(\mathrm{E}^{* *}\right)$ we can find an associated sequence of solutions to $\left(\mathrm{AIP}_{\varepsilon}^{\Pi}\right)$.

Concretely, one can examine the energies $\mathcal{E}$ and $\mathcal{E}_{\infty}$ from the last subsection (see (6.3) and (6.2)) once again to realize that $\mathcal{E}_{\infty}$ is the relaxation of $\mathcal{E}$. Again, our results are applicable and show reverse approximability of the relaxed problems by approximate solutions to the non-relaxed problems.

In the terminology of the relaxation theory for rate-independent problems as introduced in $[17,18]$, we have shown the lower incremental relaxation condition. Such a conditions has previously been seen to hold in the special case of the theory of phase-transitions in elastic solids [31]. See also [15,20] for models where a gradient Young-measure relaxation is employed.

\section{References}

[1] F. Auricchio and L. Petrini, Improvements and algorithmical considerations on a recent three-dimensional model describing stress-induced solid phase transformations. Int. J. Numer. Meth. Engng. 55 (2002), 1255-1284. 
[2] F. Auricchio and U. Stefanelli, Well-posedness and approximation for a onedimensional model for shape memory alloys. M3AS: Math. Models Meth. Appl. Sci. 15 (2005), 1301-1327.

[3] G. Bouchitté, A. Mielke, and T. Roubíček, A complete-damage problem at small strains. ZAMP Z. Angew. Math. Phys. (2009). In press, Online First. WIAS preprint 1228.

[4] A. Braides, Г-convergence for beginners, volume 22 of Oxford Lect. Series Math. Appl. Oxford University Press, 2002.

[5] C. Carstensen,, K. Hackl, and A. Mielke, Non-convex potentials and microstructures in finite-strain plasticity. Proc. Royal Soc. London, Ser. A 458 (2002), 299-317.

[6] P. Colli and A. Visintin, On a class of doubly nonlinear evolution equations. Comm. Partial Differential Equations 15 (1990)(5), 737-756.

[7] B. Dacorogna, Direct Methods in the Calculus of Variations. Springer Verlag, Berlin, 1989.

[8] G. Dal Maso, An introduction to $\Gamma$-convergence. Birkhäuser Boston Inc., Boston, MA, 1993.

[9] G. Dal Maso, G. Francfort and R. Toader, Quasistatic crack growth in nonlinear elasticity. Arch. Rational Mech. Anal. 176 (2005), 165-225.

[10] M. Frémond, Non-Smooth Thermomechanics. Springer Verlag, Berlin, 2002.

[11] G. Francfort and J. J. Marigo, Revisiting brittle fracture as an energy minimization problem. J. Mech. Phys. Solids 46 (1998), 1319-1342.

[12] G. Francfort and A. Mielke, Existence results for a class of rate-independent material models with nonconvex elastic energies. J. reine angew. Math. 595 (2006), 55-91.

[13] B. Halphen and Q. S. Nguyen, Sur les matériaux standards généralisés. J. Mécanique 14 (1975), 39-63.

[14] W. Han and B. D. Reddy, Plasticity (Mathematical Theory and Numerical Analysis), Volume 9 of Interdisciplinary Applied Mathematics. Springer Verlag, New York, 1999.

[15] M. Kružík, A. Mielke, and T. Roubíček, Modelling of microstructure and its evolution in shape-memory-alloy single-crystals, in particular in CuAlNi. Meccanica 40 (2005), 389-418.

[16] A. Mainik and A. Mielke, Existence results for energetic models for rate-independent systems. Calc. Var. PDEs 22 (2005), 73-99.

[17] A. Mielke, Evolution of rate-independent inelasticity with microstructure using relaxation and Young measures. In: C. Miehe, editor, IUTAM Symposium on Computational Mechanics of Solid Materials at Large Strains (Stuttgart Aug. 2001). Kluwer, 2003, 33-44.

[18] A. Mielke, Deriving new evolution equations for microstructures via relaxation of variational incremental problems. Comput. Methods Appl. Mech. Engrg. 193 (2004), 5095-5127.

[19] A. Mielke, Evolution in rate-independent systems (Ch. 6). In: C. Dafermos and E. Feireisl, editors, Handbook of Differential Equations, Evolutionary Equations, vol. 2, 461-559. Elsevier B.V., Amsterdam, 2005.

[20] A. Mielke and T. Roubíček, A rate-independent model for inelastic behavior of shapememory alloys. Multiscale Model. Simul. 1 (2003), 571-597. 
[21] A. Mielke and T. Roubíček, Numerical approaches to rate-independent processes and applications in inelasticity. M2AN Math. Model. Numer. Anal. (2009). In press. WIAS Preprint 1169.

[22] A. Mielke and F. Theil, A mathematical model for rate-independent phase transformations with hysteresis. In: H. D. Alber, R. Balean and R. Farwig, (eds.), Proceedings of the Workshop on "Models of Continuum Mechanics in Analysis and Engineering". Shaker Verlag, Aachen, 1999, 117-129.

[23] A. Mielke and F. Theil, On rate-independent hysteresis models. Nonl. Diff. Eqns. Appl. (NoDEA) 11 (2004), 151-189. (Accepted July 2001).

[24] A. Mielke, T. Roubíček and U. Stefanelli, $\Gamma$-limits and relaxations for rateindependent evolutionary problems. Calc. Var. PDEs 31 (2008), 387-416.

[25] A. Mielke, F. Theil and V. I. Levitas, A variational formulation of rate-independent phase transformations using an extremum principle. Arch. Rational Mech. Anal. 162 (2002), 137-177.

[26] S. Müller, Singular perturbations as a selection criterion for minimizing sequences. Calc. Var. Partial Diff. Eqns. 1 (1993), 169-204.

[27] S. Müller, Variational models for microstructure and phase transitions. In: Calculus of Variations and Geometric Evolution Problems (Cetraro, 1996), pp. 85-210. Springer, Berlin, 1999.

[28] M. Ortiz and E. Repetto, Nonconvex energy minimization and dislocation structures in ductile single crystals. J. Mech. Phys. Solids 47 (2) (1999), 397-462.

[29] F. Rindler, Optimal control for nonconvex rate-independent evolution processes. SIAM J. Control Optim. 47 (6) (2008), 2773-2794.

[30] F. Rindler, Approximation of rate-independent optimal control problems. In preparation.

[31] F. Theil, Relaxation of rate-independent evolution problems. Proc. Roy. Soc. Edinburgh Sect. A 132 (2002), 463-481.

[32] H. Ziegler and C. Wehrli, The derivation of constitutive relations from the free energy and the dissipation function. In: Advances in Applied Mechanics, Vol. 25, pp. 183237. Academic Press, Orlando, FL, 1987.

\author{
Alexander Mielke \\ Mohrenstraße 39 \\ 10117 Berlin \\ Germany \\ and \\ Mathematisches Institut \\ Humboldt Universität zu Berlin \\ Rudower Chaussee 25 \\ 12489 Berlin \\ Germany \\ e-mail: mielke@wias-berlin.de
}

Weierstraß Institut für Angewandte Analysis und Stochastik 
Filip Rindler

Technische Universität Berlin

Institut für Mathematik, Sekr. 2-2

Straße des 17. Juni 136

10623 Berlin

Germany

e-mail: rindler@math.tu-berlin.de

Received: 14 November 2007.

Accepted: 27 October 2008. 